\title{
A potent SARS-CoV-2 neutralising nanobody shows therapeutic efficacy in the Syrian golden hamster model of COVID-19
}

Jiandong Huo (10 1,2,3, Halina Mikolajek ${ }^{4}$, Audrey Le Bas1,2,3, Jordan J. Clark (1D 5, Parul Sharma5, Anja Kipar ${ }^{5,6}$, Joshua Dormon 1,3, Chelsea Norman 1,3, Miriam Weckener ${ }^{1}$, Daniel K. Clare ${ }^{4}$, Peter J. Harrison 3,4, Julia A. Tree ${ }^{7}$, Karen R. Buttigieg7 , Francisco J. Salguero7, Robert Watson (10 7, Daniel Knott7, Oliver Carnell (1) 7,

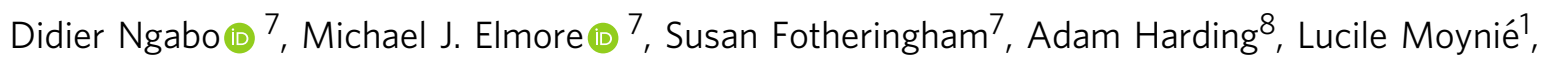
Philip N. Ward (1) 2,3, Maud Dumoux (1) 1, Tessa Prince (1) 4, Yper Hall7, Julian A. Hiscox 4,9,10, Andrew Owen (1) 11, William James (10 ${ }^{8}$, Miles W. Carroll7,12, James P. Stewart (1D ${ }^{4,9,13}$, James H. Naismith (1) ${ }^{1,2,3 凶}$ \& Raymond J. Owens (iD) 1,2,3凶

SARS-CoV-2 remains a global threat to human health particularly as escape mutants emerge. There is an unmet need for effective treatments against COVID-19 for which neutralizing single domain antibodies (nanobodies) have significant potential. Their small size and stability mean that nanobodies are compatible with respiratory administration. We report four nanobodies ( $C 5, \mathrm{H} 3, \mathrm{C} 1, \mathrm{~F} 2)$ engineered as homotrimers with pmolar affinity for the receptor binding domain (RBD) of the SARS-CoV-2 spike protein. Crystal structures show $\mathrm{C} 5$ and $\mathrm{H} 3$ overlap the ACE2 epitope, whilst C1 and F2 bind to a different epitope. Cryo Electron Microscopy shows $\mathrm{C} 5$ binding results in an all down arrangement of the Spike protein. $\mathrm{C} 1, \mathrm{H} 3$ and $\mathrm{C} 5$ all neutralize the Victoria strain, and the highly transmissible Alpha (B.1.1.7 first identified in Kent, UK) strain and C1 also neutralizes the Beta (B.1.35, first identified in South Africa). Administration of $\mathrm{C} 5$-trimer via the respiratory route showed potent therapeutic efficacy in the Syrian hamster model of COVID-19 and separately, effective prophylaxis. The molecule was similarly potent by intraperitoneal injection.

\footnotetext{
${ }^{1}$ Structural Biology, The Rosalind Franklin Institute, Harwell Science Campus, Didcot, UK. ${ }^{2}$ Division of Structural Biology, The Wellcome Centre for Human Genetics, University of Oxford, Oxford, UK. ${ }^{3}$ Protein Production UK, The Rosalind Franklin Institute - Diamond Light Source, The Research Complex at Harwell, Science Campus, Didcot, UK. ${ }^{4}$ Diamond Light Source Ltd, Harwell Science Campus, Didcot, UK. ${ }^{5}$ Department of Infection Biology \& Microbiomes, Institute of Infection, Veterinary and Ecological Sciences, University of Liverpool, Liverpool, UK. ${ }^{6}$ Laboratory for Animal Model Pathology, Institute of Veterinary Pathology, Vetsuisse Faculty, University of Zurich, Zurich, Switzerland. ${ }^{7}$ National Infection Service, Public Health England, Porton Down, Salisbury, UK. ${ }^{8}$ Sir William Dunn School of Pathology, University of Oxford, Oxford, UK. ${ }^{9}$ Department of Preventive Veterinary Medicine, Northwest A\&F University, Yangling, Shaanxi, China. ${ }^{10}$ Infectious Diseases Horizontal Technology Centre (ID HTC), A*STAR, Singapore, Singapore. ${ }^{11}$ Department of Pharmacology and Therapeutics, Centre of Excellence in Long-acting Therapeutics (CELT), University of Liverpool, Liverpool, UK. ${ }^{12}$ Nuffield Department of Medicine, University of Oxford, Oxford, UK. ${ }^{13}$ Department of Infectious Disease, University of Georgia, Georgia, USA.

$凶_{\text {email: james.naismith@strubi.ox.ac.uk; ray.owens@strubi.ox.ac.uk }}$
} 
$\mathrm{T}$ here are currently seven known coronaviruses that infect humans of which three (SARS-CoV-1, MERS and SARSCoV-2) have emerged in the last 20 years and caused severe and even fatal respiratory diseases ${ }^{1}$. By far the most serious outbreak has been caused by SARS-CoV-2 which is responsible for the current global pandemic currently associated with 3.94 million deaths worldwide. Although vaccines are now being administered against SARS-CoV-2, building up immunity in the global population will take time. The imperative to treat SARS$\mathrm{CoV}-2$ infection has led to the search for agents that neutralise the virus for use in passive immunotherapy. Early attention has focused on identifying neutralising monoclonal antibodies from patients who have recovered from COVID-192-6; the therapeutic use of antibodies is widespread and draws on existing knowledge and resources. However, nanobodies or VHHs (Variable Heavychain domains of Heavy-chain antibodies) derived from the heavy chain-only subset of camelid immunoglobulins offer an alternative with multiple advantages over conventional antibodies. The small molecular size and stability of nanobodies allows them to be formulated for topical delivery directly to the airways of infected patients through aerosolization. This results in improved bioavailability, simpler therapeutic compliance and easier administration. Secondly, while conventional antibodies that comprise two disulphide-linked polypeptides, heavy and light chain, typically require mammalian cells for production, nanobodies can be manufactured using readily available microbial systems. The potency of nanobodies against SARS-CoV- $2^{7}$ infection has been demonstrated in cell-based assays ${ }^{8-16}$ and most recently in animal studies ${ }^{17,18}$. Several strategies for engineering $\mathrm{VHH}$ into a multivalent species are known. These include fusing to an $\mathrm{Fc}^{17,19-21}$ and simple $\mathrm{N}$ to $\mathrm{C}$ fusion of two or more nanobodies to the same epitope ${ }^{19,22}$. Multivalent presentations increase the binding avidity to the molecular target and thus the biological potency of such agents ${ }^{23}$. We have isolated four nanobodies that bind different epitopes on the receptor binding domain (RBD) of the SARS-CoV-2 spike (S) glycoprotein with high affinity and potently neutralise the virus in vitro with picomolar potency. We have explored their binding to and neutralisation of two newly emergent variants (B.1.1.7 and B.1.351), identifying a potent cross-reactive agent. We have shown that treatment either systemically (intraperitoneal route) or via the respiratory tract (intranasal route) with a single dose of the most potent nanobody prevented disease progression in the Syrian hamster model of COVID-19.

\section{Results}

Isolation and binding characterisation of nanobodies that block ACE2 binding to the Spike protein of SARS-CoV-2. Antibodies to the RBD of SARS-CoV-2 were raised in a llama by primary immunisation with a combination of purified RBD alone and RBD fused to human IgG1, followed by a single boost with purified $S$ (spike) protein mixed with RBD. The $S$ protein sequence was derived from the original Wuhan or Victoria (B) strain of SARS-CoV-2. A phage display VHH library was constructed from the cDNA of peripheral blood mononuclear cells, and RBD binders selected by two rounds of bio-panning. The phage clones with the highest affinity for RBD were identified by an inhibition ELISA and classified by sequencing of complementary determining region 3 (CDR3) (Supplementary Fig. 1). Four VHHs were selected for production and their RBD-binding kinetics measured by surface plasmon resonance (SPR) (Fig. 1a-d). The calculated $K_{\mathrm{D}} \mathrm{s}$ were all in the picomolar range $(20-615 \mathrm{pM})$ with the rank order of affinities $\mathrm{H} 3>\mathrm{F} 2>\mathrm{C} 5 \gg \mathrm{C} 1$ (Table 1).
Competition binding experiments were carried out by SPR to investigate whether the VHHs blocked the binding of RBD to ACE2 and the overlap with the epitope recognised by the human monoclonal antibody CR3022 24 as well as the nanobody H11$\mathrm{H} 4{ }^{25}$. The results showed that $\mathrm{C} 1, \mathrm{H} 3$ and $\mathrm{C} 5$ blocked ACE2 binding whereas F2 did not affect ACE2 binding (Fig. 1e). C1 and $\mathrm{F} 2$ but not $\mathrm{C} 5$ or $\mathrm{H} 3$ competed with CR3022 for binding to the RBD (Fig. 1f) whereas $\mathrm{C} 5$ and $\mathrm{H} 3$ but not $\mathrm{C} 1$ and $\mathrm{F} 2$ competed with H11-H4 binding (Fig. 1g). (CR3022 is known to recognise an epitope that does not overlap with $\mathrm{ACE} 2^{25-27}$ or $\mathrm{H} 4-\mathrm{H} 11^{25}$ ). $\mathrm{C} 5$ and $\mathrm{H} 3$ would be expected to target a similar epitope to that of H11-H4, human monoclonal antibodies and other nanobodies that neutralise SARS-CoV-2 by competing directly with the interaction between the spike protein and the ACE2 receptor (cluster 2 antibodies ${ }^{28}$ ). C1 and F2 belong to the group of antibodies (cluster 1 antibodies ${ }^{28}$ ) including CR3022 26 and EY$6 \mathrm{~A}^{29}$ that bind to a region distinct from the ACE2 receptorbinding interface. These two antibodies have been reported to destabilise the trimeric spike protein and by this mechanism prevent receptor engagement ${ }^{26,29}$ thereby neutralising the virus.

ITC was used to analyse the binding of C5, F2 and C1 to RBD and spike proteins in solution However, as the agents bind so tightly conventional ITC has large errors. Therefore a displacement assay was devised using the H11 nanobody previously identified $^{25}$ that weakly binds to RBD with a $K_{D}$ of $1 \mu \mathrm{M}$ measured by ITC (Supplementary Fig. 2a). Combining the H11 titration with viral proteins (Supplementary Fig. 2a, b), C5 titration with viral proteins (Supplementary Fig. 2c, d) and C5 titration with viral proteins pre-incubated with $\mathrm{H} 11$ (displacement assay Supplementary Fig. 1e, f), we determined $K_{\mathrm{D}}$ for C5 to $\mathrm{RBD}$ as $210 \pm 60 \mathrm{pM}$ and to Spike as $350 \mathrm{pM} \pm 6 \mathrm{pM}$ (Supplementary Fig. 1g, h). The estimated $K_{\mathrm{D}}$, confirms sub-nanomolar binding of $\mathrm{C} 5$ to the Spike protein in solution and indicates 1:1 stoichiometry. No displacement agent was available for F2 and $\mathrm{C} 1$, and therefore the binding $K_{\mathrm{D}}$ for RBD of $320 \pm 30$ and $600 \pm 40$ pM respectively were estimated by direct binding but are subject to considerable uncertainty (Supplementary Fig. 1i, j). Both $\mathrm{C} 1$ and F2 when bound to Spike gave complex traces, suggesting that when engaging the Spike other conformational changes occur (Supplementary Fig. 1i, j).

The four nanobodies were also assessed for their binding to RBD from the Alpha (B.1.1.7; N501Y originally identified from the $\mathrm{UK}$ ) and Beta (B.1.351; N501Y, N417K and E484K, originally identified from South Africa). C5 and H3 bound strongly to the Alpha variant albeit with reduced affinity compared to the Victoria strain (Fig. 1h, i) however, no binding was detected to the Beta strain. By contrast, $\mathrm{C} 1$ and F2 bound with a similar affinity to all three strains (Fig. 1). These results are consistent with the C5 and $\mathrm{H} 3$ epitopes overlapping with the mutated regions which are known to be adjacent to and part of the ACE2 binding region.

Structural analysis of RBD binding. To further define the epitopes recognised by the nanobodies, crystal structures of the C5RBD (Victoria), H3-C1-RBD (Victoria) and F2-RBD (Victoria) co-complexes were determined to high resolution (Tables 2, 1.5, 1.9 and $2.3 \AA$, respectively), however, the C1-RBD binary complex failed to give high quality crystals. Examination of the three structures confirmed the results of binding experiments that indeed $\mathrm{H} 3$ and $\mathrm{C} 5$ occlude the RBD binding site for ACE2 (Fig. 2a). C1 does not occlude the ACE2 epitope but would sterically prevent ACE2 binding to RBD, F2 would not be predicted to interfere with ACE2 binding (Fig. 2a). The C5 epitope has only a small overlap with the $\mathrm{H} 3$ epitope or with the $\mathrm{H} 11-\mathrm{H} 4$ epitope that we previously reported ${ }^{25}$. The interface between C5 and RBD is extensive and involves all three CDR loops and the 
a

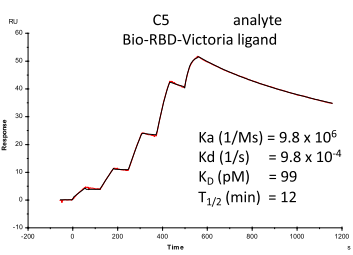

c

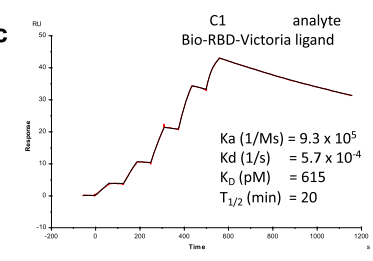

b

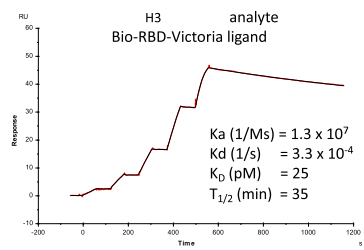

d

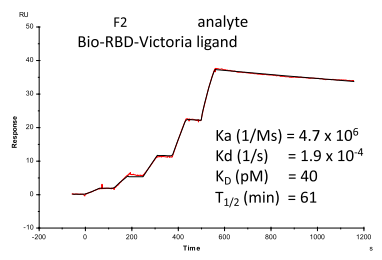

e

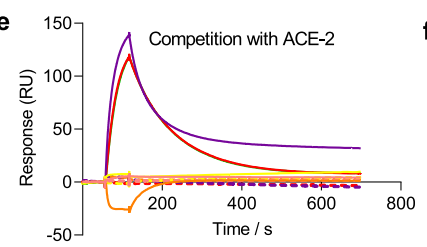

$\mathbf{g}$
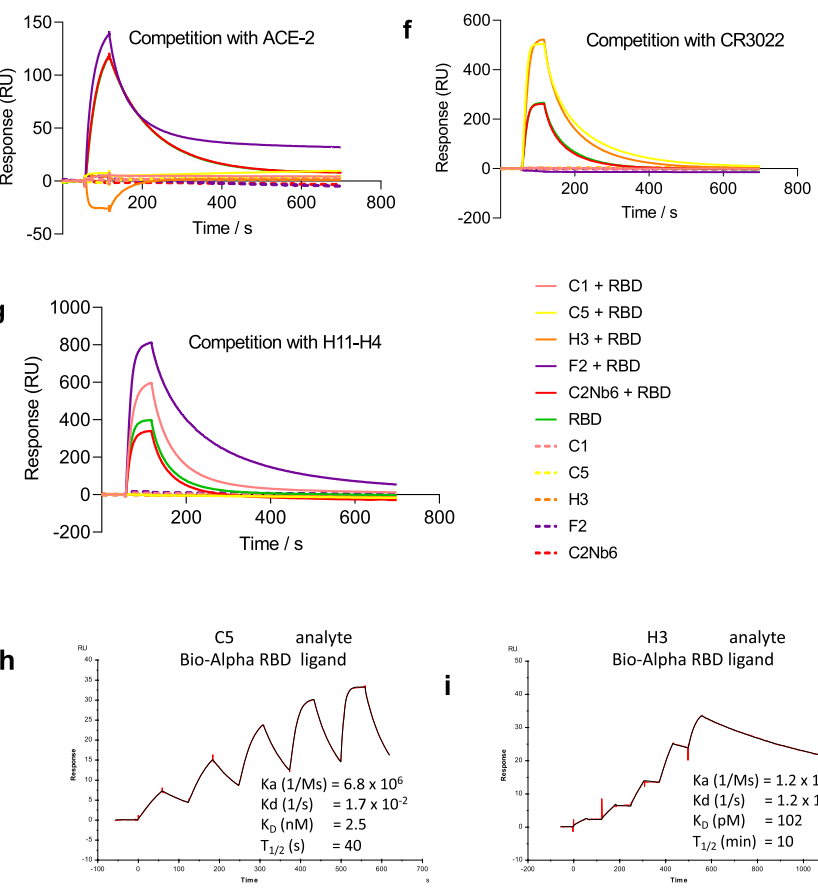

$-\mathrm{C} 1+\mathrm{RBD}$

- $\mathrm{C} 5+\mathrm{RBD}$

- $\mathrm{H} 3+\mathrm{RBD}$

- $\mathrm{F} 2+\mathrm{RBD}$

- $\mathrm{C} 2 \mathrm{Nb6}+\mathrm{RBD}$

- RBD

-.. C1

C5

-.. $\mathrm{H} 3$

-.. F2

-.. $\mathrm{C} 2 \mathrm{Nb6}$ h

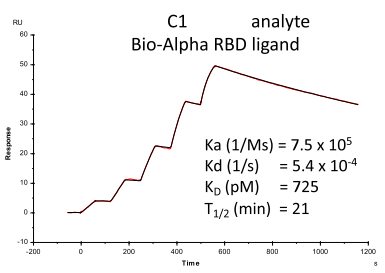

I

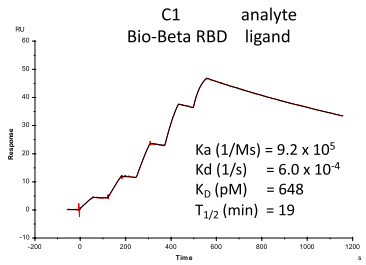

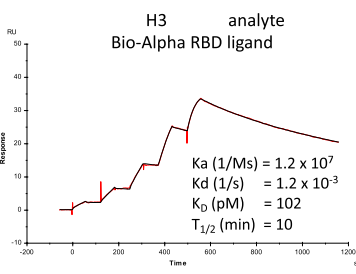

k
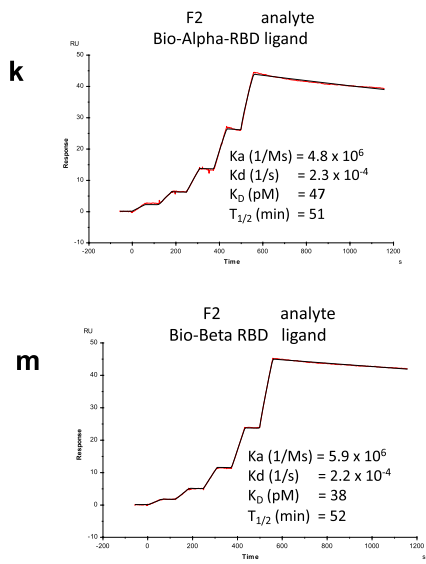

Fig. 1 Nanobody binding kinetics. a-d SPR sensorgrams showing binding kinetics of nanobody $\mathrm{C} 5, \mathrm{H} 3, \mathrm{C} 1$ and F2 for RBD Victoria (immobilised as biotinylated RBD on the chip), e- $\mathbf{g}$ SPR sensorgrams of competition assays between RBD and C5, H3, C1, F2 for binding to e ACE-2, $\mathbf{f}$ CR3022 and $\mathbf{g}$ H11$\mathrm{H} 4$, with all ligands immobilised as Fc fusion proteins and C2Nb6 (an anti-Caspr2 nanobody) used as a negative control, h-k binding kinetics of nanobody $\mathrm{C} 5, \mathrm{H} 3, \mathrm{C} 1$ and F2 to Alpha RBD (I, m) C1 and F2 binding to Beta RBD (immobilised as biotinylated RBD on the chip). 


\section{Table 1 Summary of nanobody binding kinetics.}

\begin{tabular}{|c|c|c|c|c|c|}
\hline Analyte & Ligand & Ka (1/Ms) & $K d(1 / s)$ & $K_{\mathrm{D}}(\mathrm{pM})$ & $T_{1 / 2}(\min )$ \\
\hline $\mathrm{C} 1$ & RBD & $9.3 \mathrm{E}+05$ & $5.7 \mathrm{E}-04$ & 615 & 20 \\
\hline $\mathrm{C} 1$ & Alpha RBD & $7.5 E+05$ & $5.4 \mathrm{E}-04$ & 725 & 21 \\
\hline $\mathrm{C} 1$ & Beta RBD & $9.2 \mathrm{E}+05$ & $6.0 \mathrm{E}-04$ & 648 & 19 \\
\hline $\mathrm{C} 5$ & Alpha RBD & $6.8 \mathrm{E}+06$ & 1.7E-02 & 2523 & 1 \\
\hline $\mathrm{H} 3$ & RBD & $1.3 \mathrm{E}+07$ & $3.3 \mathrm{E}-04$ & 25 & 35 \\
\hline $\mathrm{H} 3$ & Alpha RBD & $1.2 \mathrm{E}+07$ & $1.2 \mathrm{E}-03$ & 102 & 10 \\
\hline $\mathrm{F} 2$ & Beta RBD & $5.9 E+06$ & $2.2 \mathrm{E}-04$ & 38 & 52 \\
\hline $\mathrm{C} 5 \mathrm{Fc}$ & RBD & $3.1 \mathrm{E}+06$ & $1.2 \mathrm{E}-04$ & 37 & 99 \\
\hline C5 trimer & RBD-FC & $7.1 \mathrm{E}+06$ & $1.2 \mathrm{E}-04$ & 18 & 92 \\
\hline C5 trimer & Alpha RBD-Fc & $9.9 E+06$ & $2.8 \mathrm{E}-04$ & 29 & 41 \\
\hline $\mathrm{H} 3$ trimer & RBD-FC & $1.2 \mathrm{E}+08$ & 3.3E-05 & 0.3 & 349 \\
\hline $\mathrm{H} 3$ trimer & Alpha RBD-Fc & $1.8 \mathrm{E}+07$ & $1.2 \mathrm{E}-04$ & 6 & 98 \\
\hline
\end{tabular}

Table 2 X-ray crystallography data collection and refinement statistics.

\begin{tabular}{|c|c|c|c|c|c|}
\hline & C5 -RBD (70AO) & H3- C1-RBD (70AP) & F2-RBD (7OAY) & C5-Alpha RBD (70AU) & H3-C1-Alpha RBD (70AQ) \\
\hline \multicolumn{6}{|l|}{ Data collection } \\
\hline Space group & $P 2{ }_{1} 2_{2} 2$ & $P 4_{3} 2_{1} 2$ & $P 3_{1}$ & $P 2_{1}$ & $P 4_{3} 2_{1} 2$ \\
\hline \multicolumn{6}{|l|}{ Cell dimensions } \\
\hline$\alpha, \beta, \gamma\left(^{\circ}\right)$ & $90,90,90$ & $90,90,90$ & $90,90,120$ & $90,100.3,90$ & $90,90,90$ \\
\hline Resolution $(\AA)^{a}$ & $51-1.50(1.54-1.50)$ & $62-1.9(1.95-1.90)$ & $94-2.34(2.40-2.34)$ & $39-1.65(1.69-1.65)$ & $53-1.55(1.59-1.55)$ \\
\hline$R_{\text {merge }}$ & $0.045(0.39)$ & $0.124(1.83)$ & $0.156(1.75)$ & $0.104(1.29)$ & $0.100(3.12)$ \\
\hline $\mathrm{CC}_{1 / 2}$ & $1.0(0.96)$ & $0.99(0.94)$ & $1.0(0.5)$ & $1.0(0.6)$ & $1.0(0.6)$ \\
\hline Completeness (\%) & $99.4(93.7)$ & $100(100)$ & $100(99.6)$ & $100(100)$ & $100(93)$ \\
\hline Redundancy & $11.8(6.0)$ & $25.4(22.1)$ & $10.1(7.0)$ & $6.6(6.0)$ & $26.8(27.6)$ \\
\hline \multicolumn{6}{|l|}{ Refinement } \\
\hline Resolution $(\AA)$ & $46.3-1.5(1.54-1.50))$ & $62-1.9(1.95-1.90))$ & $94-2.34(2.40-2.34)$ & $39-1.65(1.69-1.65)$ & $53-1.55(1.59-1.55)$ \\
\hline No. reflections & $51,782(3353)$ & $50,644(3478)$ & $91,842(4643)$ & $77,705(5819)$ & $93,033(6677)$ \\
\hline Water & 290 & 235 & 323 & 470 & 375 \\
\hline \multicolumn{6}{|l|}{ Residual $B$ factors } \\
\hline Protein & 28 & 28 & 36 & 18 & 39 \\
\hline Ligand/ion & 44 & 71 & - & 43 & 46 \\
\hline Water & 38 & 45 & 48 & 37 & 41 \\
\hline \multicolumn{6}{|l|}{ R.m.s. deviations } \\
\hline Bond lengths $(\AA)$ & 0.008 & 0.010 & 0.009 & 0.007 & 0.008 \\
\hline Bond angles $\left({ }^{\circ}\right)$ & 1.4 & 1.52 & 1.72 & 1.34 & 1.40 \\
\hline
\end{tabular}

fixed sequence loop (FR2) at A75 of the nanobody (Fig. 2b and Supplementary Fig. 3a).

The epitopes recognised by $\mathrm{H} 3$ and $\mathrm{H} 11-\mathrm{H} 4$ as we hypothesised do have a significant overlap (Fig. 3a). H3 however has 100fold higher affinity than H11-H4. Since H3 and H11-H4 have quite different sequences and this results from many small changes in loops between the structure. This means that the identification of the atomic features that drive the difference in affinity from simple structural analysis is not straightforward. Comparison of the structures reveals several features that may contribute to the increased affinity The H3-RBD interface buries just under $10 \%$ more surface area and satisfies 4 more hydrogen bonds than in H11-H4 RBD. In addition, in H3 the key R52 E484 salt bridge makes additional hydrophobic interactions with W53 and F59 of H3 (Supplementary Fig. 3b), these contacts are absent in $\mathrm{H} 11-\mathrm{H} 4$. In a future study, we suggest these regions should be probed.

The key binding interaction between $\mathrm{C} 5$ and $\mathrm{H} 3$ nanobodies and RBD is a combined salt bridge $\pi$-cation interaction involving an arginine from the nanobody (R31 in C5, R52 in H3) with E484 
a

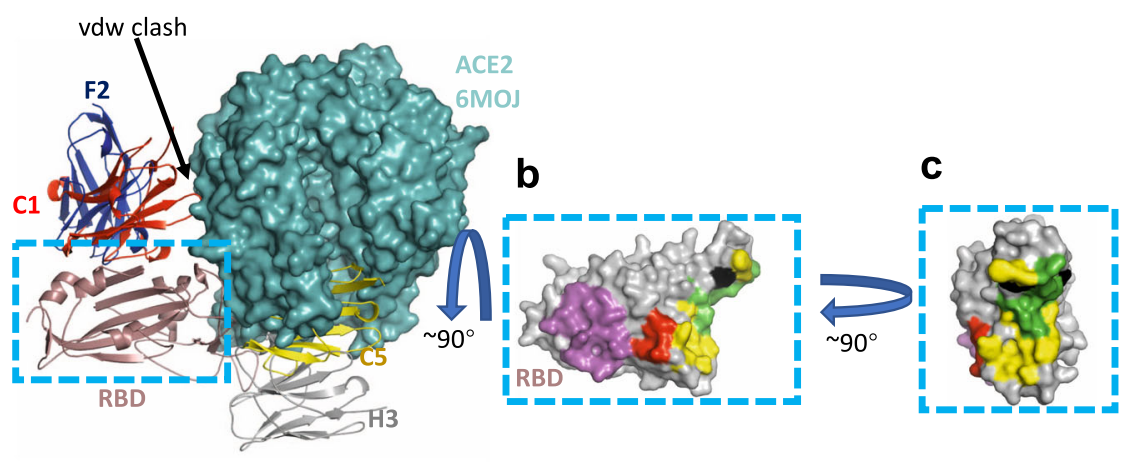

d

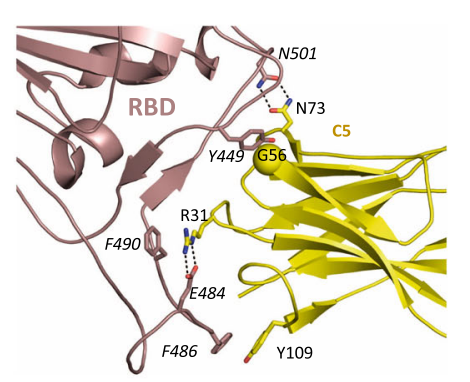

f

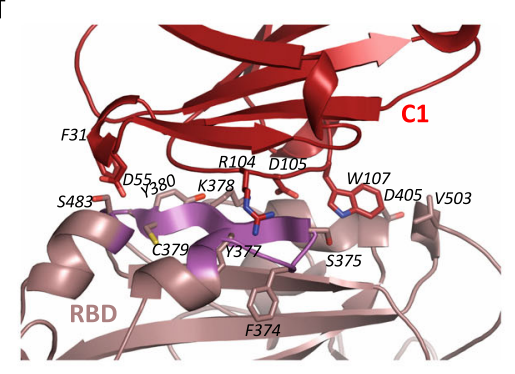

h

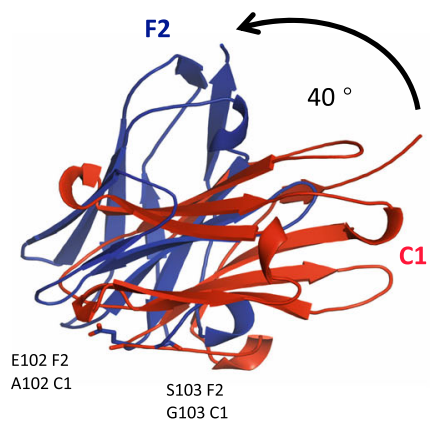

e

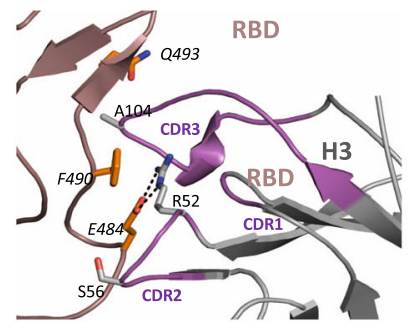

g

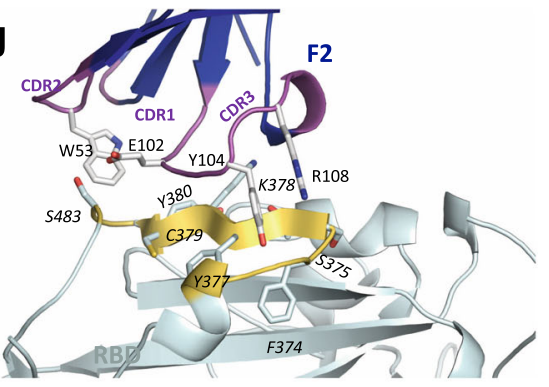

i

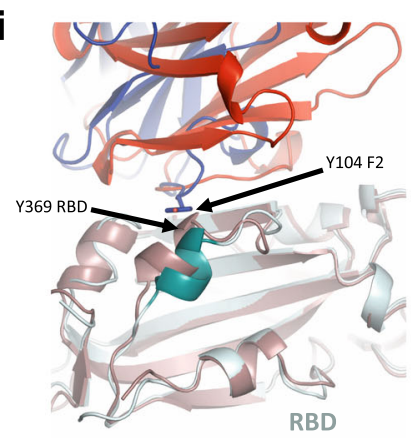

Fig. 2 Crystal structures of nanobody-RBD complexes. a The four nanobodies of this study are shown in cartoon and labelled. The figure was generated by superimposing the RBD protein from each crystal structure, only one RBD monomer is shown. Also shown is ACE2 (cyan surface) from the RBD ACE2 complex (PDB 6MOJ), positioned by superposition of the RBD. Nanobodies C5 and H3 compete with ACE2 for binding to RBD. F2 and C1 bind to a different epitope, although a loop of C1 (G42) would clash with ACE2 (arrow). b RBD is shown as a surface, the RBD molecule has been rotated by $90^{\circ}$ relative to $\mathbf{a}$. The surface is coloured magenta corresponds to the epitope engaged by both $\mathrm{C} 1$ and F2, in red is the additional region recognised by $\mathrm{C} 1$ only. In yellow is the epitope recognised by $\mathrm{C} 3$ only, in black by $\mathrm{H} 3$ only and in green by both $\mathrm{C} 5$ and $\mathrm{H} 3$. $\mathbf{c}$ The same molecule and colour scheme as b but rotated by $90^{\circ}$ to more clearly show the $\mathrm{H} 3$ and $\mathrm{C} 5$ epitopes. The key molecular interactions between $\mathbf{d} \mathrm{C} 5, \mathbf{e ~ H 3}, \mathbf{f} \mathrm{C} 1$, and $\mathbf{g}$ F2 and RBD are identified and labelled. RBD is in approximately in the same orientation as $\mathbf{a}$. In $\mathbf{f}$ and $\mathbf{g}$ coloured in magenta and gold respectively is the portion of RBD that is also recognised by both C1 and F2. h C1 and F2 bind to RBD in different orientations and overlap at residues 102 and 103. Their spatial relationship can be described as an approximate $40^{\circ}$ rotation around the main chain at 102 and 103. $\mathbf{i}$ In the F2 (blue) RBD (cyan) complex, Y102 of F2 results in a displacement of the helix at Y369 of RDB relative to the $\mathrm{C} 1$ (red) and RBD (brown) complex. The orientation of the molecules are the same as shown in Fig. 2a. All structural figures were prepared using PyMOL (http://www.pymol.org/). 

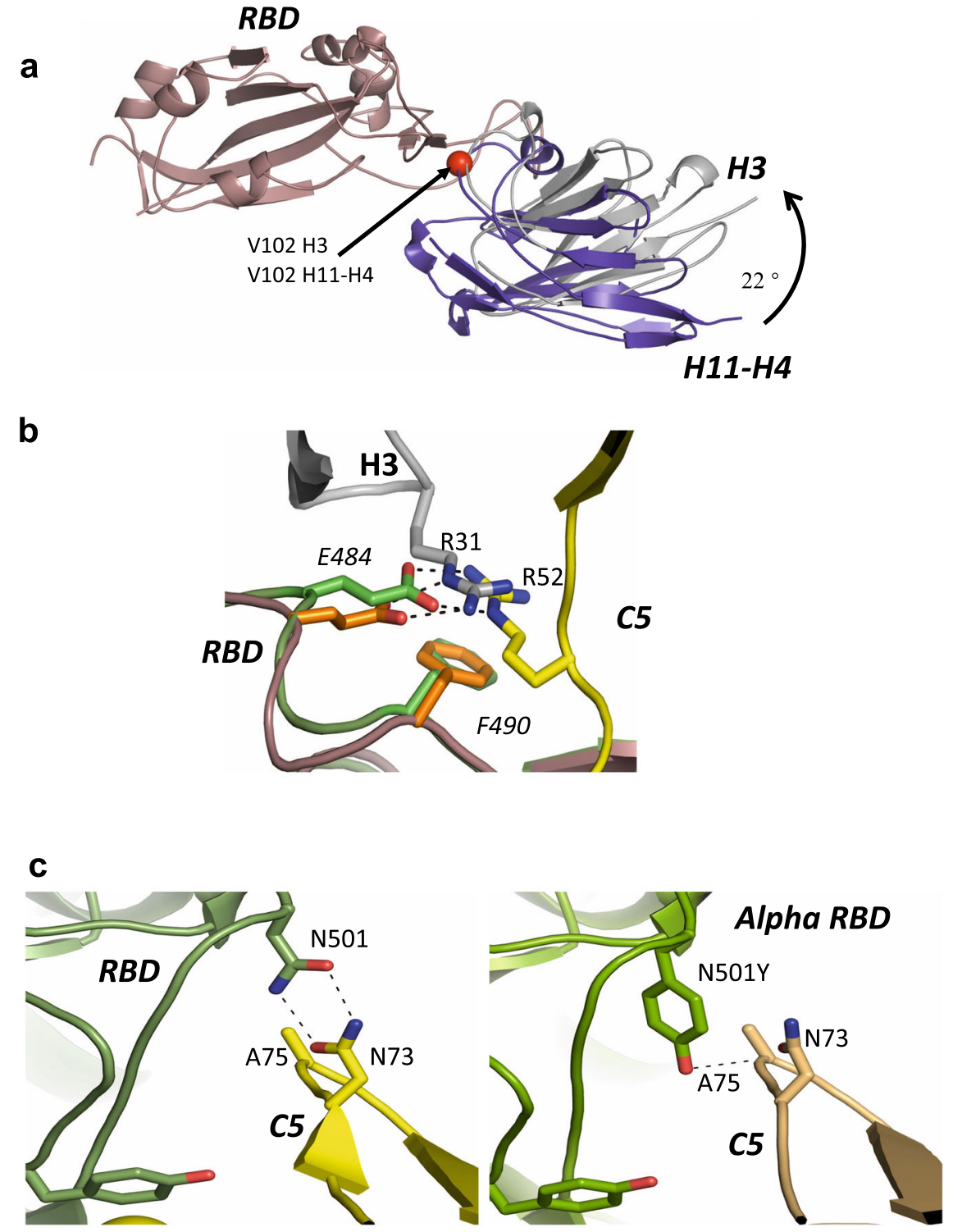

Fig. 3 Comparison of nanobody-RBD complexes. a Superimposition of H11-H4-RBD and H3-RBD complexes; V102 is shown by a red sphere. b Overlay showing the key salt bridge interaction between E484 in RBD and R31 in nanobody H3 and R52 in nanobody C5, respectively. c Close-up of the RBD-C5 interfaces for complexes with the Victoria strain of SARS-CoV-2 (N501: left hand side) and Alpha strain (N501Y: right hand side) showing the hydrogen bonding between N501 and Y501 of RBD (coloured green) with N73 of C5 in yellow and wheat respectively. Key residues are shown in stick representations.

and F490 of RBD. This arrangement of the positively charged guanidine group, phenyl ring and glutamate was previously highlighted in the H11-H4 study ${ }^{25}$. In C5, R31 is located in CDR1 and as result the side chain of R31 enters the salt bridge $\pi$-cation interaction from the opposite side to R52 but preserves the interaction (Fig. 3b). The E484K mutation found in the recently emergent South African and Brazilian strains will disrupt this interface in both $\mathrm{C} 5$ and $\mathrm{H} 3$ (as well as H11-H4). The formation of a salt bridge with E484 is a feature of many antibodies isolated from the B cells of COVID-19 convalescent and vaccinated individuals and escape mutants at this position are obviously a major concern for the efficacy of current vaccines ${ }^{30,31}$.

In addition to R31, residues T28 to G30 from CDR1 of C5 are also in contact with residues Y453, L455, Q493 and S494 of RBD (Fig. 2b and Supplementary Fig. 3a). The aromatic ring of Y449 of the RBD makes extensive hydrophobic contacts with the main chain residues, T53 to G56 from CDR2 of C5. From C5 FR2 the main chain of S72, the side chains of N73 and N74 make hydrogen bonds with the side chains of Q498, N501 and the main chain of S494 respectively. The bidentate hydrogen bonding arrangement of N73 (from C5) with N501 explains why this interaction is sensitive to the N501Y mutation (Alpha variant). FR2 of C5 makes van der Waal interactions with Y449 and Y495 to G496 of the RBD. Finally, CDR3 residues V100, Y109 and F110 in C5 make van der Waals contacts with E484 to F486 of RBD (Fig. $2 \mathrm{~b}$ and Supplementary Fig. 3a).

In $\mathrm{H} 3$, in addition to the $\mathrm{R} 52$ salt bridge, residues in CDR2 (R52 - F59) make either (or both) hydrogen bonds and van der Waals contacts with RBD (residues T470-I472, G482-E484 and F490) (Fig. 2c and supplementary Fig. 3a). From CDR3, I101 to Y106 make either (or both) hydrogen bonds and van der Waals contacts with RBD (Y449, L455, F456, E484, Y489, F490, 
L492-S494). Compared to the H11-H4 interaction, $\mathrm{H} 3$ has pivoted around V102 resulting in a shift of $2 \AA$ at R52. It is this pivot that brings FR2 of $\mathrm{H} 3$ into contact with RBD (Fig. $2 \mathrm{~b}$ and Supplementary Fig. 3a).

Based on the structure, the $\mathrm{H} 3$ interaction would not be expected to be sensitive to the mutation (N501Y) (Fig. 2c). The observation of the lower affinity of $\mathrm{H} 3$ for Alpha RBD is therefore surprising. In order to investigate this further the crystal structures of both $\mathrm{H} 3$ and $\mathrm{C} 5$ in complex with the Alpha RBD were determined. In neither the H3-RBD or H3-Alpha RBD complex is there any direct contact with residue 501. The crystal structures of these complexes do not reveal any differences in the nanobody RBD interface that result from the mutation. Molecular dynamics studies have identified that this mutation alters the dynamics of RBD and leads to an increase in affinity for ACE2 $2^{32}$. It may be that altered dynamics are responsible for modifying the binding of H3. In the C5-Alpha RBD complex, N73 still makes a hydrogen bond interaction with Y501 but the arrangement is less geometrically ideal than with N501, consistent with the lower binding affinity observed (Fig. 3c).

The $\mathrm{RBD}$ epitopes recognised by $\mathrm{C} 1$ and F2 substantially overlap (Y369-A372, F374-T385 in common) but are not identical (Fig. 2a, f, g and Supplementary Fig. 3c, d). The C1 and F2 nanobodies are oriented differently, the relationship can be described as an approximate $40^{\circ}$ rotation around residues 102 and 103 of CD3 (Fig. 2h). Interestingly this is very similar pivot point as we observed between $\mathrm{H} 3$ and H11-H4 (Fig. 3a). C1 buries more surface area and engages with several residues that are not contacted by F2 (G404-D405, V407, V503-G504 and Y508). F2 meanwhile contacts L368, P412-Q414 and D427-E429 that are not engaged by C1. C1 relies mainly on CDR3 (R100W107, S109-S110 and D112) with some contact with CDR2 (W50, S52, S54, D55 and T57-T59) and one interaction with CDR1 (F31). The same regions are employed by F2 and once again CDR3 dominates (D99-Y105, R108, T110, E11 and E113) followed by CDR2 (S52, W53, T56, P57 and Y59) and one residue in CDR1 (T28). Comparing the RBD structures in the various complexes shows that Y104 of F2 displaces the helix of RBD at Y369 by $3 \AA$ (Fig. 2i).

Residues T376- T385 of RBD also form part of the binding site of the $\mathrm{VH}$ domain of CR3022 26 . Koenig et al. ${ }^{11}$ very recently reported two anti-RBD nanobodies (VHH_V and VHH_U) that bind in a similar location to $\mathrm{C} 1$ (and F2) and target this epitope (residues Y369-K378). On repeated passage of SARS-CoV-2 escape mutations were observed at these interface residues $(\mathrm{Y} 369 \mathrm{H}, \mathrm{S} 371 \mathrm{P}, \mathrm{F} 377 \mathrm{~L} \text { and } \mathrm{K} 378 \mathrm{Q} / \mathrm{N})^{11}$, however actual variants incorporating these changes have yet to be identified ${ }^{33}$.

In the context of the whole virus and from ultrastructural analysis of purified Spike by cryo-EM, RBD exists in an equilibrium of up and down conformations. Interaction between the spike protein and cell-surface ACE2 requires at least one RBD in the up or open conformation ${ }^{34,35}$. The cryoEM structure of the C5 bound to the spike protein (stabilised in the prefusion state ${ }^{34}$ ) was determined by single particle cryoEM (Table 3 and Supplementary Figs. 4 and 5). C5 nanobodies were observed bound to the 3 down (inactive) ${ }^{36}$ form of the spike trimer (Fig. 4a). Simple modelling shows that C5 (unlike H11-H4) is unlikely to bind to the 1 up 2 down active form due to steric clashes (Fig. 4b). We conclude that although C5 can only bind to the all down of the Spike, dynamic equilibrium between Spike conformers, results in the conversion to the all down complex. Other nanobody bound spike complexes have shown binding to either both up and down $\mathrm{RBDs}^{12}$ or only up conformations ${ }^{11}$. Incubation of $\mathrm{C} 1$ or $\mathrm{F} 2$ with the trimeric spike protein led to ill-defined aggregates on
EM grids, indicating they destabilise the trimer, which would disrupt ACE2 engagement (Supplementary Fig. 4). Similar findings were reported for CR3022 $2^{26}$ and EY-6A ${ }^{29}$ that recognise this epitope and are consistent with the complex ITC traces observed for binding of $\mathrm{C} 1$ and F2 to the spike protein in solution (Supplementary Fig. 2) This was attributed to the epitope being in the middle of the molecule and binding of a protein to this epitope is incompatible with the trimeric Spike structure.

Potent neutralisation of SARS-CoV-2 in vitro by trimeric nanobodies. Linking more than one nanobody together to create bivalent and trivalent assemblies significantly increases antigenbinding due to avidity ${ }^{11,13,23,37-39}$. Therefore, trivalent versions of the four nanobodies were constructed by joining the VHH domains with a glycine-serine flexible linker, $(\mathrm{GS})_{6}$. The nanobody homo-trimers ( $\mathrm{C} 5, \mathrm{Cl}$ and $\mathrm{H} 3$ ) were produced by transient expression in expi293 cells and purified by metal chelate affinity chromatography and size exclusion. Although the F2 trimer was expressed it proved to be unstable on purification and was not pursued further. Binding of the trimeric nanobodies to the RBD was measured by SPR, and an approximate 10 to 100 -fold enhancement in $K_{\mathrm{D}}$ was observed compared to the monomers (Table 1 and Supplementary Fig. 6). Notably, the $\mathrm{H} 3$ trimer was shown to have a sub-picomolar $K_{\mathrm{D}}$ for the RBD-Victoria with an off rate of $\sim 6 \mathrm{~h}$. Binding of C5 trimer to RBD-Kent was shown to be only two-fold weaker than to RBD-Victoria, whilst binding of

Table 3 EM statistics.

\begin{tabular}{ll|} 
& \\
& Spike C5 (PDB ID 7OAN, \\
EMD-12777)
\end{tabular}


a

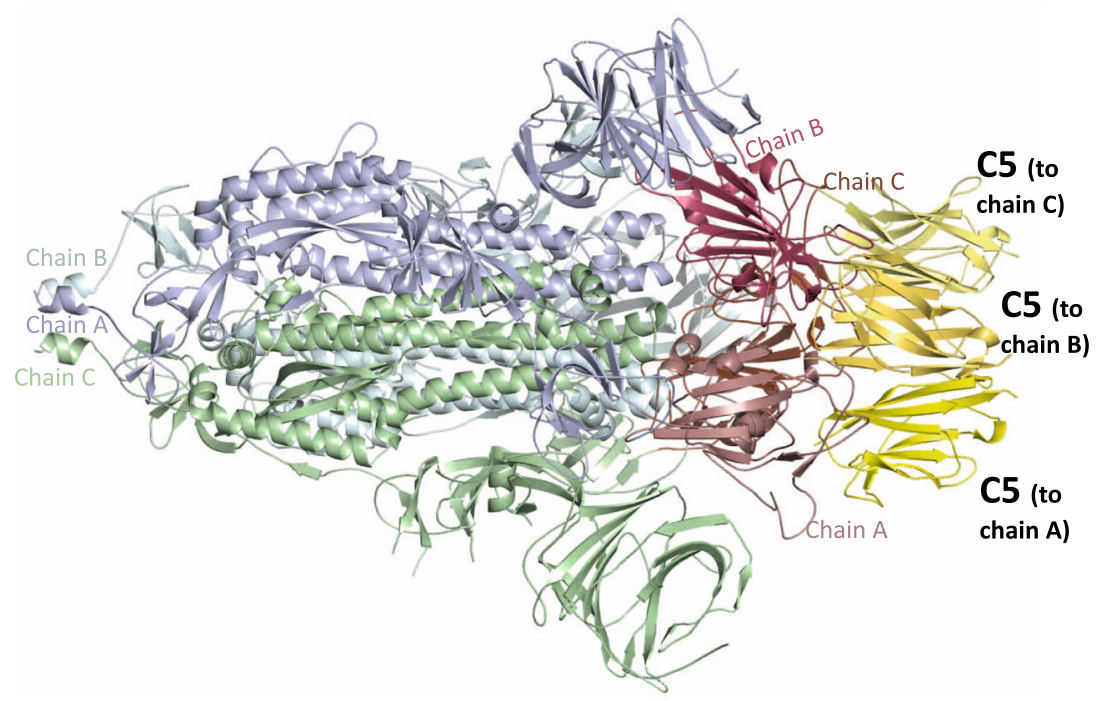

b

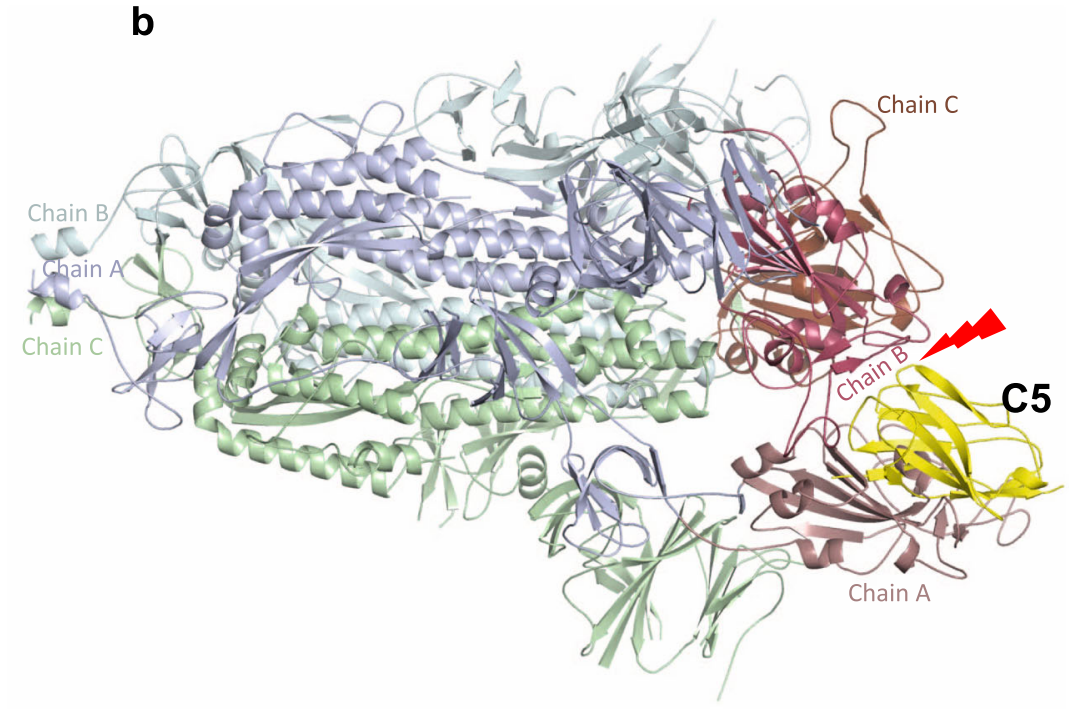

Fig. 4 Cryo-EM structure of C5-Spike complex. a EM structure of spike (S1) trimer with each of three chains bound to one C5 nanobody coloured yellow. The other spike monomers are coloured pale cyan, green and purple wheat and throughout and show that all three RDBs are in the down conformation. b Superimposition of $\mathrm{C} 5$ onto the spike protein in the two down one up conformation shows that there would be significant clashes that would prevent this interaction.

C5 monomer was 25 -fold weaker (Table 1, Fig. 1 and Supplementary Fig. 6).

Microneutralisation assays were carried out to test the effectiveness of the three nanobody trimers to block infection of Vero E6 cells by either Victoria, Alpha or Beta strains of the virus. All nanobodies potently neutralised some if not all the strains (Fig. 5). Although $\mathrm{H} 3$ bound more tightly than $\mathrm{C} 5$ to the RBDs in vitro, it was less potent than $\mathrm{C} 5$ against both Victoria and Beta strains (Fig. 5b). Crucially, C5 was equipotent in neutralising these strains with IC50s of $18 \mathrm{pM}$ (Victoria - B) and $25 \mathrm{pM}$ (Alpha B1.1.7) (Fig. 5b). As anticipated from the in vitro binding data, only $\mathrm{C} 1$ was active against the Beta (B1.351) strain (Fig. 5c).

The neutralisation potency of the $\mathrm{C} 5$ trimer was confirmed in the Gold Standard Plaque Reduction Neutralization Test (PRNT) against the Victoria strain which gave an ND50 of $3 \mathrm{pM}$ (Supplementary Fig. 7). This corresponds to one of the most potent neutralising nanobodies that has been identified to date $^{10,13,39,40}$ and was therefore chosen to test for efficacy in an animal model of COVID-19.
C5-Fc fusion shows therapeutic efficacy in vivo. To probe neutralisation in vivo, we tested $\mathrm{C} 5$ in the Syrian hamster model of COVID-1941-43. As first demonstrated with SARS-CoV ${ }^{44}$, Syrian hamsters are readily infectable, display both upper and lower respiratory tract viral replication, clinical signs and also pathological changes that are similar to those seen in infected humans. Since an anti-MERS-CoV nanobody fused to immunoglobulin Fc fragment has previously shown to extend the halflife of the protein in vivo and ameliorate disease in a mouse challenge model ${ }^{45}$ we first tested $\mathrm{C} 5$ as a huIgG1 Fc fusion protein. The RBD-binding affinity $\left(K_{\mathrm{D}} 37 \mathrm{pM}\right)$ and virus neutralisation potency (ND50 of $2 \mathrm{pM} ; 180 \mathrm{pg} / \mathrm{ml}$ ) of $\mathrm{C} 5-\mathrm{Fc}$ was similar to the trivalent $\mathrm{C} 5$ protein, confirming the importance of multivalency for effective neutralisation (Table 1 and Supplementary Figs. 6 and 7). Efficacy of a human IgG1 antibody has also been demonstrated in the Syrian hamster model with the isotype matched control showing no therapeutic effect ${ }^{6}$.

The study comprised an experimental and a control group each of six animals. All animals in both groups were challenged 
Victoria (BVIC01)

a

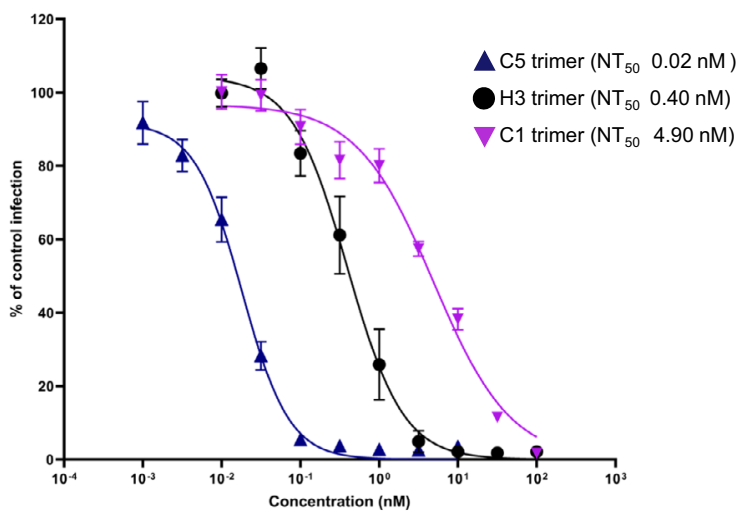

Alpha (B.1.1.7)

b
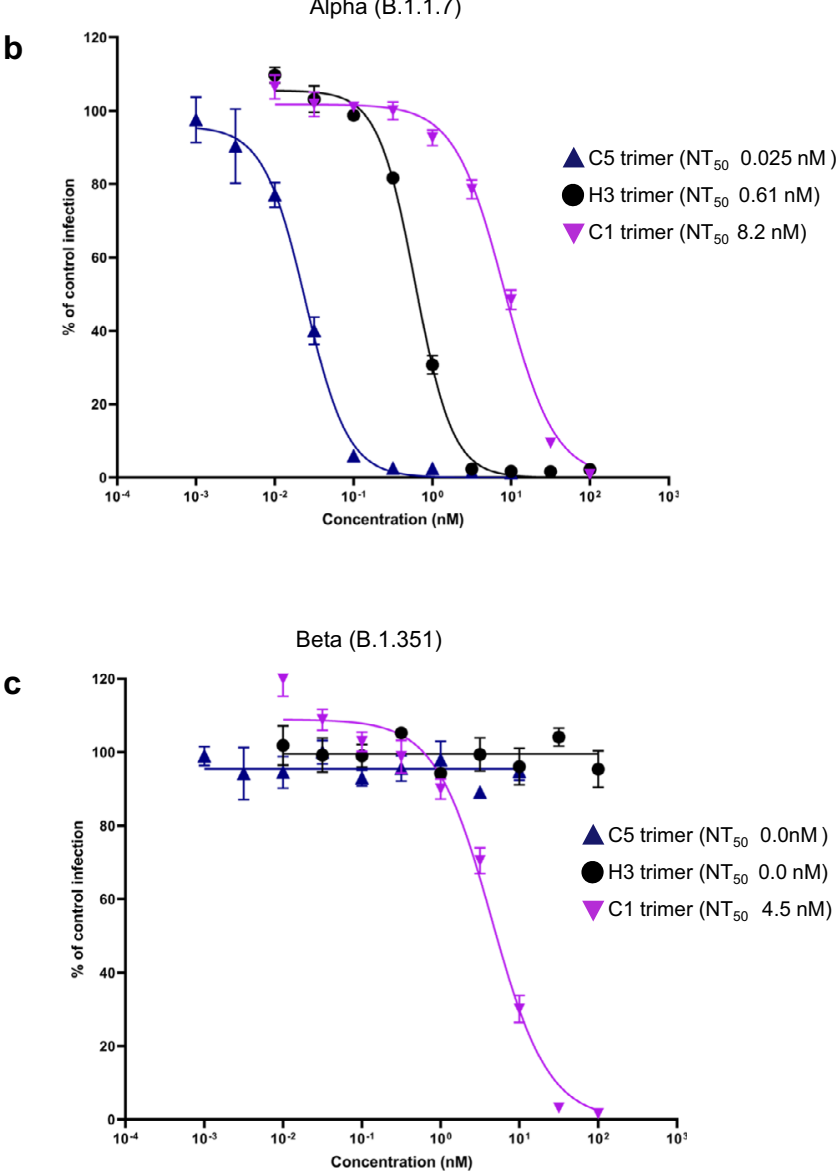

Fig. 5 Neutralisation of SARS-CoV-2 strains in vitro. Neutralisation curves of the anti-RBD nanobody trimers for a Victoria (BVIC01), b Alpha, (B1.1.7) and c Beta, (B1.351) strains of SARS-CoV-2 measured in a microneutralisation assay. Data are shown as the mean $(n=4) \pm 95 \% \mathrm{Cl}$.

intranasally (IN) with SARS-CoV-2 Victoria $\left(5 \times 10^{4} \mathrm{pfu}\right)$. The experimental group was treated $24 \mathrm{~h}$ later with a single dose of C5-Fc (4 mg/kg) administered intraperitoneally (IP) whilst the control group were left untreated (Fig. 6a). As a measure of disease progression, the animals were weighed each day over 7 days and nasal washes and oropharyngeal swabs were taken every other day (Fig. 6a). On day 7 the animals were culled and viral load in lung, trachea and duodenum measured by subgenomic (sg)-RT-qPCR. Vital organs were formalin-fixed for histopathology (H\&E staining) and ISH RNAScope staining with SARS-CoV-2 S-gene probe to detect presence of virus RNA.
SARS-CoV-2 infected animals exhibited progressive mean body weight loss (up to 17\%) from day 1 to day 7 post challenge (pc) (Fig. 6b). In contrast, by day 7 post challenge (pc), animals in the nanobody treated group had lost significantly $(P<0.005$, Mann-Whitney) less weight (7\%). High levels of nasal shedding of live virus $\left(10^{4}-10^{5} \mathrm{FFU} / \mathrm{ml}\right)$ were detected in $6 / 6$ untreated animals $(100 \%)$ on day 2 pc, whereas only $3 / 6(50 \%)$ animals in the nanobody treated group shed virus (Fig. 6c). Some live viral shedding was seen in the throats of $3 / 6$ control animals whereas no live virus was detected in the nanobody treated animals $(0 / 6)$ on any day (Fig. 6c). Statistically significant lower levels of viral RNA were detected in throat swabs of treated compared to untreated controls on days 2, 4 and 7 pc (Fig. 6e). However no difference in viral RNA was found in the nasal washes taken over the time course of the study or in homogenates of lung, trachea and duodenum following culling of the animals on day 7 (Fig. 6e, f). Measurements of sgRNA copies in either nasal washes, throat swabs and tissues showed no significant differences between the number of genomic copies of the virus between control and treated animals (Fig. 6d, f).

Histopathology and RNAScope ISH techniques were used to compare the pathological changes and the presence of viral RNA in tissues from nanobody-treated and untreated control hamsters. A semiquantitative scoring system was combined with digital image analysis to calculate the area of lung with pneumonia and the quantity of virus. Viral RNA and lesions consistent with infection with SARS-CoV-2 were observed only in the nasal cavity (Supplementary Fig. 8) and lungs (Supplementary Fig. 9). No lesions were observed in any other organ studied. The lung lesions consisted of a bronchointerstitial pneumonia showing areas of parenchymal consolidation and were characterised by infiltration of macrophages and neutrophils, but also some lymphocytes and plasma cells (Supplementary Fig. 8c). The lesions in the nasal cavity consisted in necrosis of the respiratory and olfactory mucosa and presence of inflammatory exudates and cell debris within the nasal cavity lumen. The area with pneumonia was significantly lower in the nanobody-treated hamsters together with a marked reduction of histopathology scores in the nasal cavity (Supplementary Fig. 9a). Statistically significant differences were also found for the presence of virus RNA in the lung or the nasal cavity (Supplementary Fig. 8b and 9b). Together, these results showed that a single therapeutic dose of C5-Fc administered IP reached the site of action in the lungs and nasal cavity and reduced viral load and associated pathological changes. Therefore, based on these promising results we undertook a larger study to evaluate the C5 trimer in the Syrian hamster model.

Trimeric C5 nanobody shows efficacy when administered via the respiratory route. The smaller molecular size of the C5trimer $(40 \mathrm{kDa})$ compared to the $\mathrm{C} 5-\mathrm{Fc}(80 \mathrm{kDa}$ plus $2 \mathrm{~N}$-linked glycans) renders the nanobody suitable for respiratory administration directly to the airways ${ }^{46}$. Previously an anti-RSV nanobody trimer had been shown to be effective in reducing viral load in a disease model following intranasal delivery ${ }^{23}$. Therefore, in the second animal study, the efficacy of the trimeric version of C5 was evaluated in the COVID-19 hamster model by administration using both IP and intranasal routes. The study consisted of five groups of six animals that were challenged with the SARS-CoV-2 strain Liverpool $\left(1 \times 10^{4} \mathrm{pfu}\right)$ on day 0 and weight changes followed over 7 days (Fig. 7a). To compare to the results obtained with the $\mathrm{C} 5-\mathrm{Fc}$, the trimer was administered IP at $4 \mathrm{mg} / \mathrm{kg}$; the same dose was delivered directly to the airways via intranasal installation (IN). A tenfold lower intranasal dose of $0.4 \mathrm{mg} / \mathrm{kg}$ of C5-trimer was also tested. As in the first study, animals in the 
a

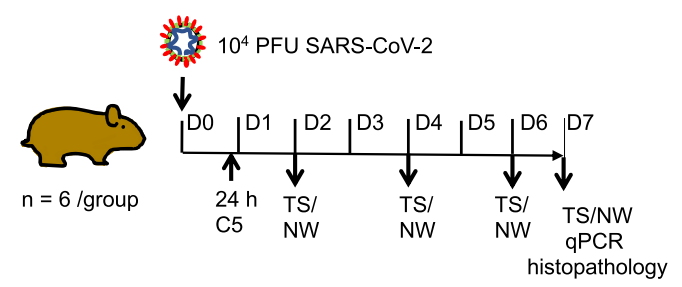

b

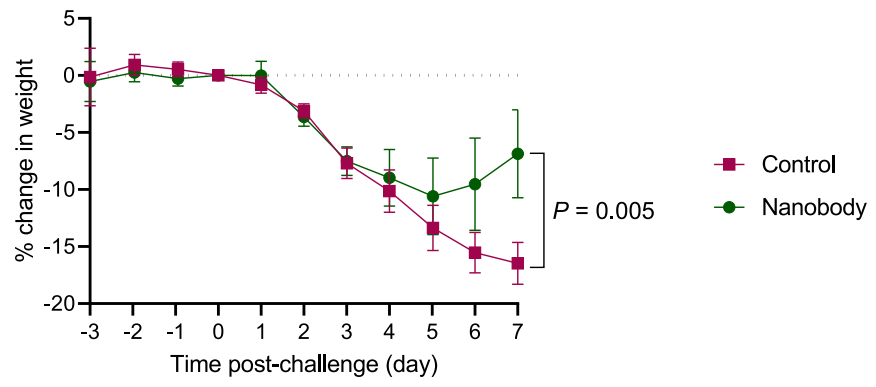

C
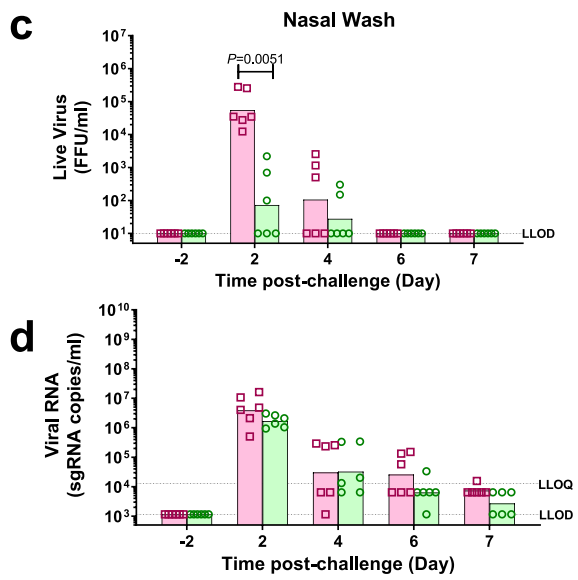

e

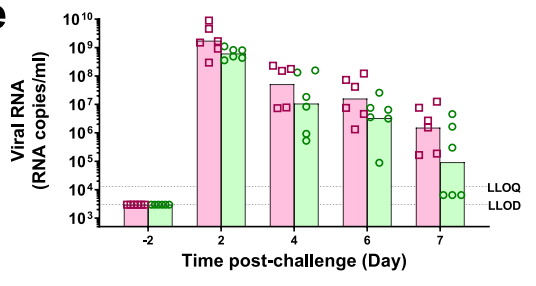

$\square$ Control $\square$ Nanobody

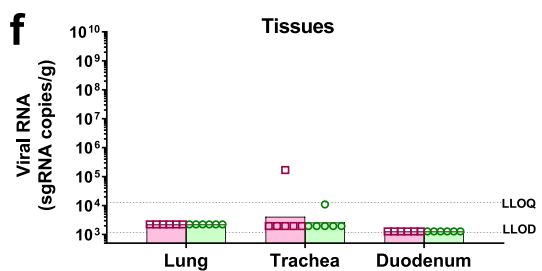

untreated group showed a significant and progressive weight loss ( $20 \%$ by day 7 ), whereas all animals treated therapeutically, $24 \mathrm{~h}$ after viral challenge, showed only a small weight loss and from day 2 had recovered to pre-challenged weights (Fig. 7b). The animals pre-treated $2 \mathrm{~h}$ before IN virus inoculation with $4 \mathrm{mg} / \mathrm{kg}$ C5 via the intranasal route showed no change in weight. The weight loss in all C5-treated groups was significantly different
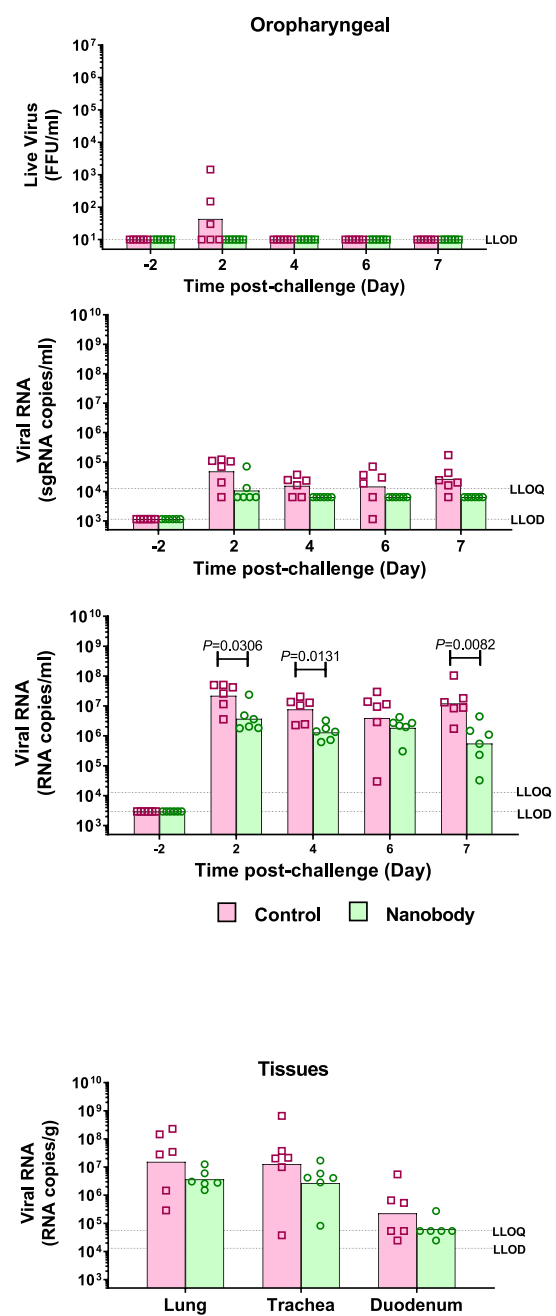

from the control group given PBS alone $(p<0.01$; repeated measures two-way ANOVA). Analysis of viral load in the postmortem lungs at day 7 by qPCR for Nucleoprotein (NP) RNA showed a decrease in the median value in treated compared to the untreated control animals. (Fig. 7c). This decrease was significantly different in the IP treated group. While there was a clear trend in the other groups, there were two outliers with 
Fig. 6 C5-Fc neutralisation of SARS-CoV-2 in the Syrian hamster model. a Golden Syrian hamsters ( $n=6$ biologically independent animals per group) were challenged with SARS-CoV-2 (B Victoria $5 \times 10^{4} \mathrm{pfu}$ ) at day 0 and then treated with either C5-Fc (IP $4 \mathrm{mg} / \mathrm{kg}$ ) or PBS, delivered by the intraperitoneal route $24 \mathrm{~h}$ post-challenge and Throat Swab (TS) and Nasal Wash (NS) samples collected on days 2, 4, 6 and 7 post virus challenge. b Body weight was recorded daily and the mean percentage weight change from baseline was plotted (mean $\pm 1 \mathrm{SE}$ ). Filled in square represents data from control animals (virus only) and filled in circles represents data from nanobody treated. Nasal washes (i-iii) and oropharyngeal swabs (iv-vi) were collected at days -2 to 2, 4, 6 and $7 \mathrm{pc}$ for all virus challenged groups. Tissue samples (lung, trachea and duodenum) were collected at post-mortem (day $7 \mathrm{pc}$ ) (vii \& viii). Open square represents data from control animals (virus only) and open circle represents data from nanobody-treated hamsters. Symbols show values for individual animals, columns represent the calculated group geometric means. c Quantitation of live virus in the nasal wash and oropharyngeal swabs using a micro-foci assay. d Number of copies of subgenomic (sg)viral RNA in the nasal wash and oropharyngeal swab. e Number of copies genomic viral RNA in the nasal wash oropharyngeal swab. $\mathbf{f}$ Number of copies of sgRNA and genomic RNA in tissues. The dashed horizontal lines show the lower limit of quantification (LLOQ) and the lower limit of detection (LLOD). The statistical test used was a Mann-Whitney's $U$ test, two-sided, using Minitab v 16

higher RNA load in each of the groups treated via the intranasal route. No live virus was detected by plaque assay in day 7 samples of lung homogenates consistent with what was observed in the first animal study (Fig. 6c).

The histological and immunohistological examination showed multifocal extensive consolidation of the lung parenchyma in the untreated group, with multifocal patches of cells that expressed viral antigen (mainly type I and II pneumocytes, some cells morphologically consistent with macrophages) (Fig. 7d). The consolidated areas contained aggregates of macrophages and some neutrophils and were otherwise comprised of activated type II pneumocytes with occasional syncytial cell formation, and hyperplastic bronchiolar epithelial cells (Supplementary Fig. 10). In all treated groups, the extent of parenchymal consolidation was substantially reduced as quantified by automated morphometric analysis which resulted in a statistically significantly larger area of ventilated lung parenchyma (Fig. 7d). The lungs of treated animals showed very limited viral antigen expression and only in occasional individual macrophages within small infiltrates or in pneumocytes in individual alveoli (Fig. 7e).

More detailed assessment of the consolidated areas in untreated animals confirmed that at day 7 post SARS-CoV-2 infection, the pathological processes in the lungs are dominated by regenerative attempts, as shown by type II pneumocyte and bronchiolar epithelial hyperplasia, in combination with macrophage dominated inflammatory infiltration (Supplementary Fig. 10). Animals that had received either C5-trimer $(4 \mathrm{mg} / \mathrm{kg})$ $2 \mathrm{~h}$ pre-infection or the lower dose $(0.4 \mathrm{mg} / \mathrm{kg})$ at $4 \mathrm{~h}$ post infection, resulted in substantially less regenerative processes; the observed small, consolidated areas were dominated by infiltrating macrophages (Supplementary Fig. 10). These findings at the late, i.e., regenerative stage of SARS-CoV-2 infection in hamsters ${ }^{42}$ indirectly confirm that the C5-trimer treatment significantly reduced pulmonary infection and induced a strong macrophage response, likely leading to phagocytosis and thereby sequestration of the virus. Double immunofluorescence for viral $\mathrm{N}$ protein and the macrophage marker Ibal undertaken on the lungs of hamsters that had been pre-treated with $\mathrm{C} 5$-trimer $2 \mathrm{~h}$ prior to virus inoculation confirmed that numerous macrophages in the focal lesions contained viral antigen (Supplementary Fig. 11).

Collectively the animal studies described herein have established that a multivalent nanobody (Fc fusion or trimer) targeted to the RBD of SARS-CoV-2 spike protein delivered either systemically or via the respiratory route has a therapeutic benefit in the hamster disease model of COVID-19. In particular, efficacy was observed with a single IN dose of $0.4 \mathrm{mg} / \mathrm{kg}$ (equating to $\sim 40 \mu \mathrm{g}$ / animal) of the C5-trimer demonstrating the high potency of this biological agent. A further dose ranging study will be required to establish the minimum amount of the nanobody required to be therapeutically effective in the hamster disease model.

\section{Discussion}

The RBD of SARS-CoV-2 is the immuno-dominant region of the virus spike protein and the target for neutralising antibodies generated either by vaccination or infection. Following immunisation of a llama with a combination of the RBD and stabilised spike trimer ${ }^{34}$ based on the Victoria strain sequence, we obtained nanobodies designated $\mathrm{C} 5, \mathrm{~F} 2, \mathrm{H} 3$ and $\mathrm{C} 1$ that bound one of two orthogonal sites on the RBD. The site recognised by $\mathrm{C} 5$ and $\mathrm{H} 3$ overlapped with the ACE2 binding site on the top surface of the domain, whilst the second recognised by $\mathrm{C} 1$ and $\mathrm{F} 2$ corresponded to a location on the side of the RBD originally identified by the SARS-CoV antibody CR3022 24,26,47 and nanobody VHH72 ${ }^{48}$. Consistent with other recent reports ${ }^{10,17,39}$ nanobodies that bound to both sites showed very potent neutralisation activity when configured as multivalent trimers, with the C5 trimer demonstrating complete inhibition of infection of Vero cells at $<$ $100 \mathrm{pM}$ in a PRNT assay. This activity was translated into a marked disease-modifying effect in the Syrian golden hamster model of COVID-19 with treated animals showing minimal weight loss and very limited pulmonary infection and associated changes following a single dose of C5 trimer $24 \mathrm{~h}$ post virus challenge. Most importantly, administration of the nanobody agent either directly by nasal administration or systemically (IP) was effective at $4.0 \mathrm{mg} / \mathrm{kg}$. Nasal administration appeared to promote faster recovery than IP perhaps reflecting increased levels of the C5 trimer reaching the sites of infection in the lungs. Recently, mice challenged intranasally with SARS-CoV-2, and then treated prophylactically IP with a nanobody Fc fusion has also been shown to reduce viral load in the lungs ${ }^{17}$. More recently, Nambulli et al. ${ }^{18}$, showed that nasal administration of a nanobody $6 \mathrm{~h}$ after viral challenge also reduced viral load and weight change in the Syrian hamster model. Our data are consistent with these results but our treatment with the C5 trimer $24 \mathrm{~h}$ after viral challenge when the clinical manifestations of disease first become apparent is a more demanding test of nanobody efficacy and arguably a more realistic model of therapeutic treatment.

The independent emergence of SARS-CoV-2 variants which appear to be more transmissible is now a major concern. Although in this study, animals were challenged with the Victoria and Liverpool (lineage B) strains, the in vitro neutralisation data strongly indicates the $\mathrm{C} 5$ trimer will be equally effective against the lineage B.1.1.7 or Alpha variant in this COVID-19 disease model. Although, the Alpha variant dominated infections in the UK in early 2021, the new Delta virus (B.1.671.2) that first 
a

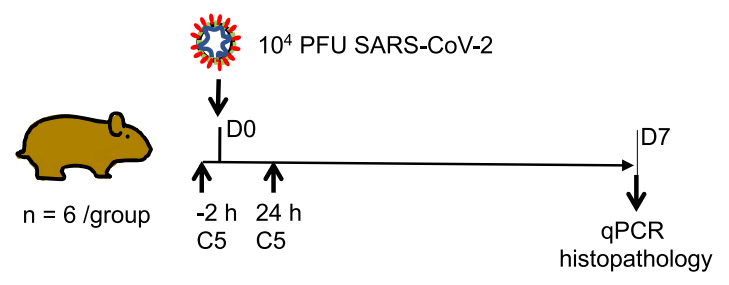

b
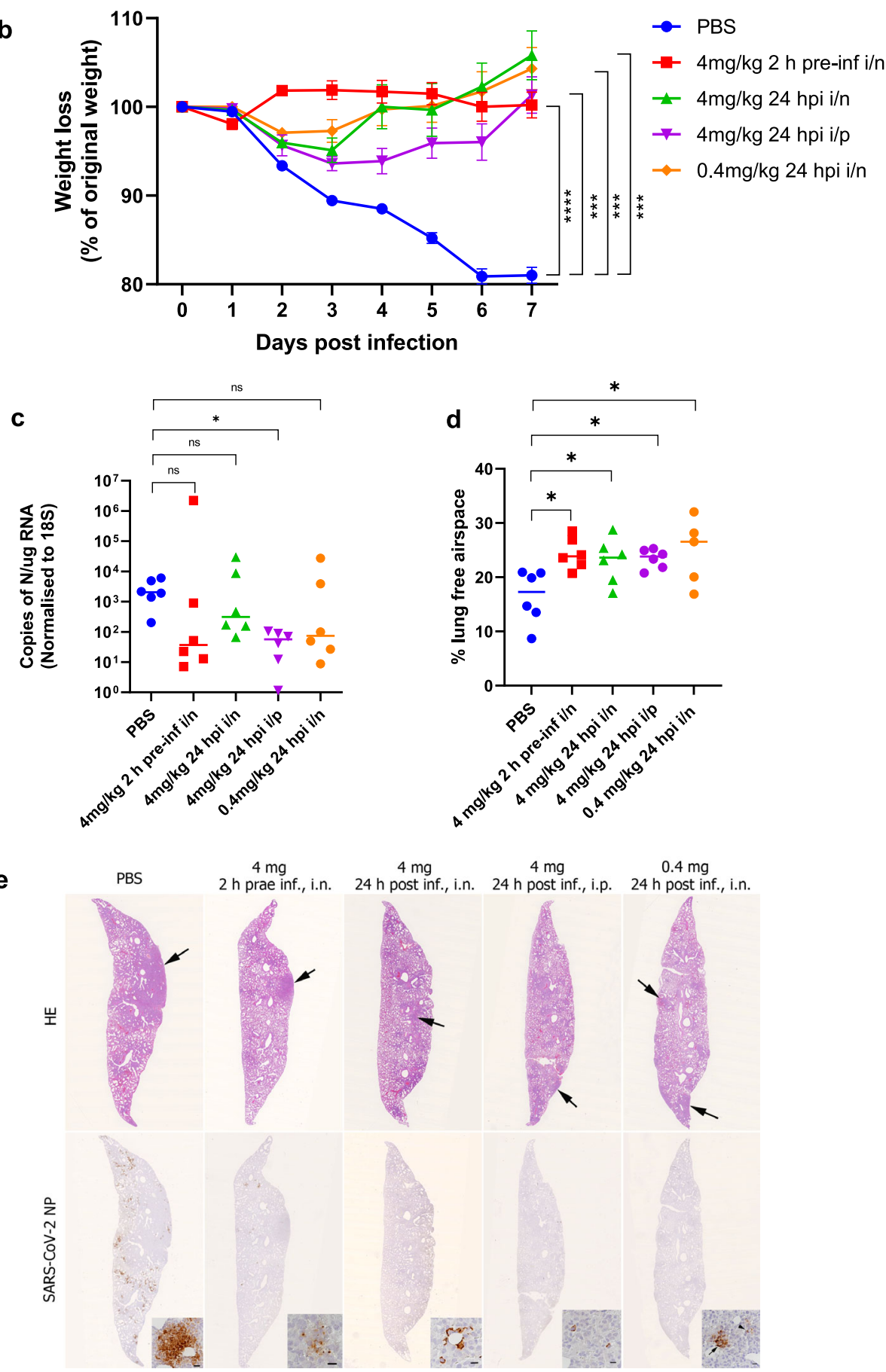

originated in India has become the most recent variant of concern. The epitope recognised by C5 does not include the two residues that are mutated in the RBD of the Delta virus, L452R and T478K. However, F54 in Framework 3 of C5 does make a Van der Waal interaction with L452 that may be disrupted by mutation to R452 (Supplementary Fig. 3). The B.1.351 (Beta variant) and P.1 (Gamma variant) lineages are characterised by three mutations (K417N, E484K and N501Y) in the RBD, which, although less prevalent, are a serious concern as they are associated with immune evasion ${ }^{30}$. Structural analysis of the C5-RBD and H3-RBD complexes showed the central importance of E484 in RBD to the interaction and unsurprisingly these nanobodies failed to neutralise the Beta virus. The $\mathrm{C} 1$ nanobody is significantly less potent than C5 against the Victoria strain, NT50 of 
Fig. 7 Therapeutic efficacy of C5 Trimer in Syrian hamster model. a Golden Syrian hamsters ( $n=6$ biologically independent animals per group) were infected intranasally with SARS-CoV-2 strain LIV (PANGO lineage B; $10^{4} \mathrm{pfu}$ ). Individual cohorts were treated either $2 \mathrm{~h}$ pre-infection or $24 \mathrm{~h}$ post-infection (hpi) with $100 \mu \mathrm{l}$ of C5 either intranasally (IN) or intraperitoneally (IP) as indicated or sham-infected with PBS. b Animals were monitored for weight loss at indicated time-points. Data are the mean value $(n=6) \pm$ SEM. Comparisons were made using a repeated-measures two-way ANOVA with GeisserGreenhouse's correction and Šídák's multiple comparisons test; at day 7: PBS vs. $4 \mathrm{mg} / \mathrm{kg} 2 \mathrm{~h}$ pre-inf i/n; ${ }^{\star \star \star \star} P<0.0001, \mathrm{PBS}$ vs. $4 \mathrm{mg} / \mathrm{kg} 24 \mathrm{hpi} \mathrm{i} / \mathrm{n}$; ${ }^{\star \star \star} P=0.0005$, PBS vs. $4 \mathrm{mg} / \mathrm{kg} 24 \mathrm{hpi} \mathrm{i/p;}{ }^{\star \star \star} P=0.0002$, PBS vs. $0.4 \mathrm{mg} / \mathrm{kg} 24 \mathrm{hpi} \mathrm{i} / \mathrm{n} ;{ }^{* \star} P=0.0003$. c RNA extracted from lungs was analysed for SARS-CoV-2 viral load using qRT-PCR for the N gene levels by qRT-PCR. Assays were normalised relative to levels of 18S RNA. Data for individual animals are shown with the median value represented by a horizontal line. Data are mean value $(n=6) \pm S E M$ and were analysed using a Kruskal-Wallis one-way ANOVA with Dunn's multiple comparisons test; PBS vs. $4 \mathrm{mg} / \mathrm{kg} 2 \mathrm{~h}$ pre-inf $\mathrm{i} / \mathrm{n} ; P=0.1682$ (ns), PBS vs. $4 \mathrm{mg} / \mathrm{kg} 24$ hpi $\mathrm{i} / \mathrm{n} ; P>0.9999$ (ns), PBS vs. $4 \mathrm{mg} / \mathrm{kg} 24 \mathrm{hpi} \mathrm{i} / \mathrm{p} ;{ }^{\star} P=0.0287, \mathrm{PBS}$ vs. $0.4 \mathrm{mg} / \mathrm{kg} 24 \mathrm{hpi} \mathrm{i} / \mathrm{n} ; P=0.4044$ (ns). d Morphometric analysis of HE-stained sections scanned and analysed using the software programme Visiopharm to quantify the area of non-aerated parenchyma and aerated parenchyma in relation to the total area. Results are expressed as the mean free airspace in lung sections. Data are mean value $(n=6) \pm$ SEM and were analysed using a one-way ANOVA with Dunnett's multiple comparisons test; PBS vs. $4 \mathrm{mg} / \mathrm{kg} 2 \mathrm{~h}$ pre-inf i $/ \mathrm{n} ;{ }^{\star} P=0.0109, \mathrm{PBS}$ vs. $4 \mathrm{mg} / \mathrm{kg} 24 \mathrm{hpi} \mathrm{i/n;}{ }^{*} P=0.0406, \mathrm{PBS}$ vs. $4 \mathrm{mg} / \mathrm{kg} 24 \mathrm{hpi} \mathrm{i} / \mathrm{p}$; ${ }^{\star} P=0.0270$, PBS vs. $0.4 \mathrm{mg} / \mathrm{kg} 24 \mathrm{hpi} \mathrm{i} / \mathrm{n} ;{ }^{\star} P=0.0110$. e Lung sections of hamsters, infected intranasally with $10^{4} \mathrm{PFU} / 100 \mu \mathrm{l}$ SARS-CoV-2 and euthanized at day 7 post infection. Animals had been untreated prior to infection (PBS) or treated with $4 \mathrm{mg} / \mathrm{kg}$ C5 IN $2 \mathrm{~h}$ prae infection (h prae inf) or $24 \mathrm{~h}$ post infection (h post inf) or IP at $24 \mathrm{~h}$ post inf, or had received $0.4 \mathrm{mg} / \mathrm{kg} \mathrm{C} 5 \mathrm{IN}$ at $24 \mathrm{~h}$ post inf. In the untreated animal (PBS) the lung parenchyma exhibits a large consolidated area (arrow) and multifocal patches with extensive viral antigen expression in particular by pneumocytes. In treated animals there are only a few small areas of consolidation (arrows). The animal treated with $4 \mathrm{mg} / \mathrm{kg} \mathrm{C} 5$ intranasally at $2 \mathrm{~h}$ prae inf exhibits a few small patches with viral antigen expression mainly in degenerate cells, all other treated animals show viral antigen expression in occasional individual macrophages within small infiltrates or in pneumocytes in individual alveoli. Top: HE stain, bottom: immunohistology for SARS-CoV-2 N, hematoxylin counterstain.

Bars $=20 \mu \mathrm{m}$ (PBS) or $10 \mu \mathrm{m}$ (all others). Images are representative $n=6$ biologically independent samples.

$\mathrm{C} 1$ trimer is $4.9 \mathrm{nM}$ compared to $18 \mathrm{pM}$ and binds to a different epitope. However, $\mathrm{C} 1$ was equally effective against all three strains of the virus tested for neutralisation in vitro, thus it has the potential to be a broadly neutralising agent.

The relative size and stability of nanobody based biotherapeutics has fuelled interest in their use as inhaled drugs for the treatment of respiratory diseases ${ }^{49}$, including for COVID$19^{50}$. Furthermore, since some of their formulations, for example the trimeric molecule discussed here, do not require mammalian cell culture, they are relatively inexpensive to produce. In laboratory tests, anti-SARS-CoV-2 nanobody trimers, similar to the ones we report here, have already been shown to be stable under aerosolisation ${ }^{10,13}$. Indeed, the trimeric anti-RSV nanobody (ALX-0171) $)^{23}$, was successfully administered using a nebuliser in a Phase 1 safety study. This provides a useful precedent for developing locally administered products to treat respiratory viral illnesses. Local administration of nanobody therapy may not only treat disease but by reducing viral load, may rapidly and substantially lower infectivity.

In summary, we have identified a set of potent neutralising SARS-CoV-2 nanobodies from an immunised llama library and mapped these onto the receptor-binding domain of the spike protein. The two epitopes correspond to those targeted by human antibodies recovered from convalescent patients pointing to their cross species immunodominance. We show that SARS-CoV-2 infection in a hamster model can be treated with a single dose of the most potent trimeric nanobody delivered either systemically or intranasally. Combinations of nanobodies that target different epitopes may improve resilience in combating new variants of the virus.

\section{Methods}

Immunisation and construction of VHH library. The SARS-CoV-2 receptorbinding domain (amino acids 330-532), SARS-CoV-2 receptor-binding domain fused to hIgG1 Fc (RBD-Fc) and trimeric spike protein (amino acids 1-1208) were produced as described by Huo et al. ${ }^{25}$. Antibodies were raised in a llama by intramuscular immunisation with $200 \mu \mathrm{g}$ of recombinant RBD and $200 \mu \mathrm{g}$ of RBDFc on day 0 , and then $200 \mu \mathrm{g}$ RBD and $200 \mu \mathrm{g} \mathrm{S}$ protein on day 28 . The adjuvant used was Gerbu LQ\#3000. Blood $(150 \mathrm{ml})$ was collected on day 38. Immunisations and handling of the llama were performed under the authority of the project license PA1FB163A. Peripheral blood mononuclear cells were prepared using Ficoll-Paque PLUS according to the manufacturer's protocol; total RNA was extracted using TRIzol $^{\text {Tx }}$; reverse transcription and PCR was carried out with SuperScript IV
Reverse Transcriptase using primer CALL_GSP. The pool of VHH encoding sequences were amplified by two rounds of PCR using CALL_001 and CALL_02 (round 1), VHH_For and VHH_Rev_IgG2 plus VHH_Rev_IgG3 (round 2). Following purification by agarose gel electrophoresis, the $\mathrm{VHH}$ cDNAs were cloned into the SfiI sites of the phagemid vector pADL-23c. In this vector, the VHH encoding sequence is preceded by a pelB leader sequence followed by a linker, His6 and cMyc tag (GPGGQHHHHHHGAEQKLISEEDLS). Electro-competent E. coli TG1 cells were transformed with the recombinant $\mathrm{pADL}-23 \mathrm{c}$ vector resulting in a VHH library of $\sim 4 \times 10^{9}$ independent transformants. The resulting TG1 library stock was then infected with M13K07 helper phage to obtain a library of VHHpresenting phages.

Isolation of VHHs. Phages displaying VHHs specific for the RBD of SARS-CoV-2 were enriched after two rounds of bio-panning on $50 \mathrm{nM}$ and $2 \mathrm{nM}$ of biotinylated RBD respectively, through capturing with Dynabeads ${ }^{\mathrm{Tx}} \mathrm{M}-280$ (Thermo Fisher Scientific). Enrichment after each round of panning was determined by plating the cell culture with 10-fold serial dilutions. After the second round of panning, 93 individual phagemid clones were picked, VHH displaying phages were recovered by infection with M13K07 helper phage and tested for binding to RBD by a combination of competition and inhibition ELISAs. In these assays, RBD was immobilised on a 96-well plate and binding of phage clones was measured in the presence of excess soluble RBD (inhibition ELISA) or the RBD-binding H11-H4 $\mathrm{Fc}^{25}$ (competition ELISA). Bound phage were detected with an HRP-coupled antiM13 antibody (1:5000; Cytiva 27-9421-01).

Phage binders were ranked according to the inhibition assay and then classified as either competitive with H11-H4 (i.e., sharing the same epitope) or noncompetitive (i.e. binding to a different epitope on RBD). Clones were sequenced and grouped according to CDR3 sequence identity.

Construction of trivalent VHHs. To generate the trimeric VHHs, the $\mathrm{C} 1, \mathrm{C} 5, \mathrm{H} 3$ and F2 gene fragments were used as templates to amplify three fragments by PCR with the following pairs of primers: TriNb_Neo_F1 and TriNb_R1; TriNb_F2 and TriNb_R2; TriNb_F3 and TriNb_Neo_R1; the three fragments were then joined together with a PCR reaction using primers TriNb_Neo_F2 and TriNb_Neo_R2. The trimeric gene product was then inserted into the pOPINTTGneo vector by Infusion ${ }^{\circledR}$ cloning. pOPINTTG contains a mu-phosphatase leader sequence and C-terminal His6 tag ${ }^{51}$.

Construction of receptor-binding domain variants. To generate the Alpha RBD, using the RBD-WT as template, the gene was firstly amplified as two fragments with pairs of primers (1) TTGneo_RBD_F and N501Y_R and (2) TTGneo_RBD_R and N501Y_F; the two fragments were then joined together with a PCR reaction using primer TTGneo_RBD_F and TTGneo_RBD_R. The Alpha RBD gene product was then cloned into the pOPINTTGneo vector by Infusion ${ }^{\circledR}$ cloning.

The Beta RBD gene was generated using the Alpha RBD as a template, in two steps. Firstly, two fragments were amplified with pairs of primers of (1) TTGneo_RBD_F and E484K_R and (2) TTGneo_RBD_R and E484K_F. The two fragments were then joined together with a PCR reaction using primer TTGneo_RBD_F and TTGneo_RBD_R and cloned into the pOPINTTGneo vector 
by Infusion ${ }^{\circledR}$ cloning. Secondly, using this plasmid as a template, the two fragments were amplified with pairs of primers of (1) TTGneo_RBD_F and K417V_R and (2) TTGneo_RBD_R and K417V_F. The fragments were then joined together with a PCR reaction using primer TTGneo_RBD_F and TTGneo_RBD_R and the resulting gene product was then cloned into the pOPINTTGneo vector by Infusion ${ }^{\circledast}$ cloning to produce the Beta-RBD expression vector.

To generate the huIgG1 Fc-fusion versions of RBDs, the RBD genes from the pOPINTTGneo vector were amplified by a pair of primers TTGneo_RBD_F and RBD_Fc_R, followed by being cloned into the pOPINTTGneo-Fc vector by Infusion ${ }^{\circledast}$ cloning. The pOPINTTGneo-Fc contains a mu-phosphatase leader sequence, a huIgG1 $\mathrm{Fc}$ and $\mathrm{C}$-terminal His6 tag ${ }^{51}$.

Protein production. In general, the monovalent VHHs were cloned into the vector pOPINO ${ }^{52}$ containing an OmpA leader sequence and C-terminal His6 tag. The C5 and $\mathrm{H} 3 \mathrm{VHH}$ constructs used for the crystallisation of C5-Kent RBD and H3-Kent RBD complexes, respectively, were generated through amplification with a pair of primers PelB_F and PelB_R, followed by being cloned into the phagemid vector pADL-23c by Infusion ${ }^{\circledR}$ cloning. pADL-23c contains a PelB leader sequence and C-terminal His6 tag. The plasmids were transformed into the WK6 E. coli strain and protein expression induced by $1 \mathrm{mM}$ IPTG grown overnight at $20^{\circ} \mathrm{C}$. Periplasmic extracts were prepared by osmotic shock and VHH proteins purified by immobilised metal affinity chromatography (IMAC) using an automated protocol implemented on an ÄKTXpress followed by a Hiload 16/60 Superdex 75 or a Superdex 75 10/300GL column, using phosphate-buffered saline (PBS) pH 7.4 buffer. The C5-Fc was produced by transient expression in expi293 ${ }^{\circledR}$ cells and

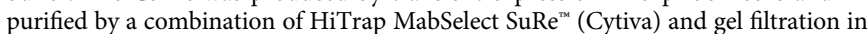
PBS pH 7.4 buffer. The trimeric versions of the nanobodies were produced by transient expression in expi293 ${ }^{\circledR}$ cells and purified by a combination of IMAC and gel filtration in PBS pH 7.4 buffer. For animal studies, an additional ion exchange chromatography step was introduced after the IMAC (GE, Capto $S 1 \mathrm{~mL}$ column) to lower endotoxin levels which were further reduced to $<0.1 \mathrm{EU} / \mathrm{ml}$ by passing in the final purified product through two Proteus NoEndo ${ }^{\text {mix }}$ clean-up columns (Generon, Slough, UK). Endotoxin levels were quantified using the Pierce ${ }^{\text {sx }}$ LAL Chromogenic Endotoxin Quantitation Kit (Thermofisher Scientific). Protein was concentrated to $4 \mathrm{mg} / \mathrm{ml}$ and flash frozen for storage at $-80^{\circ} \mathrm{C}$. The biotinylated and non-biotinylated RBDs, ACE2-Fc and CR3022-Fc were produced by transient expression in expi293 ${ }^{\circledR}$ cells $^{25}$. Briefly, Proteins were purified from culture supernatants $72 \mathrm{~h}$ post-transfection by immobilised metal affinity using an automated protocol implemented on an ÄKTAxpress (GE Healthcare, UK), followed by a Hiload 16/60 Superdex 75 or a Superdex 200 10/300GL column, using phosphatebuffered saline (PBS) pH 7.4 buffer.

All PCR primers used in this work are listed in Supplementary Table 1 and nanobody sequences are provided in the Supplementary Table 2. The pOPINO vectors for producing nanobodies $\mathrm{C} 1, \mathrm{C} 5, \mathrm{~F} 2$ and $\mathrm{H} 3$ have been deposited with Addgene (www.addgene.org) with IDs 171924, 171925, 171926 and 171927.

Surface plasmon resonance \& ITC. The surface plasmon resonance experiments were performed using a Biacore T200 (GE Healthcare). All assays were performed with a running buffer of PBS pH 7.4 supplemented with $0.005 \% \mathrm{vol} / \mathrm{vol}$ surfactant P20 (GE Healthcare) at $25^{\circ} \mathrm{C}$.

The competition assay was performed with a Sensor Chip Protein A (Cytiva). CR3022-Fc, ACE2-Fc or H11-H4-Fc was used as the ligand, 1000 RU of CR3022$\mathrm{Fc}, \mathrm{ACE} 2-\mathrm{Fc}$ or H11-H4-Fc was immobilised. The following samples were injected: (1) a mixture of $1 \mu \mathrm{M}$ nanobody C1/C5/H3/F2 and $0.1 \mu \mathrm{M}$ RBD-WT; (2) a mixture of $1 \mu \mathrm{M}$ C2Nb6 (an anti-Caspr2 nanobody) and $0.1 \mu \mathrm{M}$ RBD-WT; (3) $1 \mu \mathrm{M}$ nanobody $\mathrm{C} 1 / \mathrm{C} 5 / \mathrm{H} 3 / \mathrm{F} 2$; (4) $1 \mu \mathrm{M}$ C2Nb6 and (5) $0.1 \mu \mathrm{M}$ RBD-WT. All curves were plotted using GraphPad Prism 8.

To determine the binding kinetics between the SARS-CoV-2 RBD and nanobody C1/C5/H3/F2, a Biotin CAPture Kit (Cytiva) was used. Biotinylated RBDs were immobilised onto the sample flow cell of the sensor chip. The reference flow cell was left blank. Nanobody was injected over the two flow cells at a range of five concentrations prepared by serial twofold dilutions, at a flow rate of $30 \mu \mathrm{min}^{-1}$ using a single-cycle kinetics programme. Running buffer was also injected using the same programme for background subtraction. All data were fitted to a 1:1 binding model using Biacore T200 Evaluation Software 3.1.

To determine the binding kinetics between the SARS-CoV-2 RBD-WT and C5Fc, a Biotin CAPture Kit (Cytiva) was used. Biotinylated RBD was immobilised onto the sample flow cell of the sensor chip. The reference flow cell was left blank. C5-Fc was injected over the two flow cells at a single concentration of $10 \mathrm{nM}$, at a flow rate of $30 \mu \mathrm{min}^{-1}$. Running buffer was also injected using the same programme for background subtraction. All data were fitted to a 1:1 binding model using Biacore T200 Evaluation Software 3.1.

To determine the binding kinetics between the SARS-CoV-2 RBD and the trimeric nanobodies $\mathrm{C} 1 / \mathrm{C} 5 / \mathrm{H} 3$, a Sensor Chip Protein A (Cytiva) was used. The huIgG1 Fc-fusion versions of RBDs were immobilised onto the sample flow cell of the sensor chip. The reference flow cell was left blank. Trimeric nanobody was injected over the two flow cells at a single concentration of $25 \mathrm{nM}$ for $\mathrm{Cl}$ trimer, $10 \mathrm{nM}$ for C5 trimer and $10 \mathrm{nM}$ (RBD-Kent interaction) or $2.5 \mathrm{nM}$ (RBD-WT interaction) for $\mathrm{H} 3$ trimer, at a flow rate of $30 \mu \mathrm{min}^{-1}$. Running buffer was also injected using the same programme for background subtraction. All data were fitted to a 1:1 binding model using Biacore T200 Evaluation Software 3.1.

Isothermal titration calorimetry (ITC) measurements were carried out using an iTC200 and PEAQ-ITC MicroCalorimeter (GE Healthcare) at $25^{\circ} \mathrm{C}$. RBD and all nanobodies were dialysed into $\mathrm{PBS}$ and titrations into RBD were performed using 150 to $25 \mu \mathrm{M}$ of nanobody and $14-2 \mu \mathrm{M}$ RBD with the exception of Nb-H11 $(470 \mu \mathrm{M})$ and $\mathrm{RBD}(47 \mu \mathrm{M})$. For spike protein, $80-60 \mu \mathrm{M}$ nanobody were titrated into 8-6 $\mu \mathrm{M}$ spike (monomer concentration). Each experiment consisted of an initial injection of $0.4 \mu \mathrm{l}$ followed by 16-19 injections of 2-2.4 $\mu \mathrm{l}$ nanobody into the cell containing RBD or spike, while stirring at $750 \mathrm{rpm}$. For the displacement assays, $\sim 200 \mu \mathrm{M}$ of C5 nanobody was titrated into a mixture of $20 \mu \mathrm{M} \mathrm{RBD}$ and $100 \mu \mathrm{M} \mathrm{H} 11$ and $66 \mu \mathrm{M}$ C5 nanobody was titrated into a mixture of $6 \mu \mathrm{M}$ spike and $186 \mu \mathrm{M} \mathrm{H11}$. Data acquisition and analysis were performed using the Origin scientific graphing and analysis software package (OriginLab) or AFFINImeter for global fitting of the displacement assay. For the fitting of C5 and H11 into spike, the monomeric concentration of spike and a single binding mode have been used Data analysis was performed by generating a binding isotherm and best fit using the following parameters: $N$ (number of sites), $\Delta \mathrm{H}\left(\mathrm{kJmol}^{-1}\right), \Delta \mathrm{S}\left(\mathrm{JK}^{-1} \mathrm{~mol}^{-1}\right)$ and $\mathrm{K}$ (binding constant in $\mathrm{molar}^{-1}$ ). Following data analysis, $\mathrm{K}$ was converted to the dissociation constant $(\mathrm{Kd})$.

\section{Determination of the structure of VHH- RBD complexes by X-ray crystal-}

lography. Purified VHHs were mixed with de-glycosylated RBD at a molar ratio of $1.2: 1$, and the complex purified by size exclusion chromatography as described ${ }^{8}$. The optimal conditions for crystallisation of each complex were F2-RBD $0.1 \mathrm{M}$ Succinic Acid, Sodium Dihydrogen Phosphate and Glycine (SPG), pH 8, 25\% Polyethylene glycol (PEG) 1500, H3-C1-RBD and H3-C1-Alpha RBD $1.0 \mathrm{M}$ Lithium chloride, $0.1 \mathrm{M}$ Citric acid pH 4, 20\% PEG 6000 and C5-RBD $0.2 \mathrm{M}$ Sodium Acetate, 0.1 M Sodium Cacodylate pH 6.5, 30\% w/v PEG 8000 and the C5Alpha RBD 0.2 M Ammonium fluoride and 20\% PEG 3350. The protein concentrations for all complexes were $18 \mathrm{mg} / \mathrm{ml}$ except for F2-RBD, where $34 \mathrm{mg} / \mathrm{ml}$ was used. Crystals were grown at $20^{\circ} \mathrm{C}$ by sitting drop vapour diffusion method by mixing $0.1 \mu \mathrm{l}$ of protein complex (C5-RBD) with $0.1 \mu \mathrm{l}$ of reservoir; mixing $0.2 \mu \mathrm{l}$ of protein complex (F2-RBD; H3-C1-RBD) with $0.1 \mu \mathrm{l}$ of reservoir or $0.1 \mu \mathrm{l}$ of protein complex (C5-Alpha RBD; H3-C1-Alpha RBD) and $0.2 \mu \mathrm{l}$ of reservoir as stated above. Crystals were cryoprotected with $30 \%$ glycerol, cryocooled in liquid nitrogen, diffraction data collected and processed at the beamlines I03, I04 and I24 of Diamond Light Source, UK. The structures were solved by molecular replacement with PHASER ${ }^{53,54}$, as implemented in $\mathrm{CCP} 4^{55}$, using the individual components of the complex between H11-H4 RBD ${ }^{8}$ (PDB 6ZBP) as the search models. The resulting structures were rebuilt by hand using $\mathrm{COOT}^{56}$ and refined in REFMAC5 $5^{57}$ assisted by PDB-REDO ${ }^{58}$ and MOLPROBITY ${ }^{59}$. Where TLS parameters ${ }^{60}$ were employed, the different regions of the protein were identified using the TLSMD server ${ }^{61}$. Data processing and refinement statistics are given in Table 2.

Cryo-EM structures. Preparation of cryo-EM grids, data collection and processing were carried out as previously described ${ }^{8}$. Briefly, purified spike protein in $10 \mathrm{mM}$ Hepes, $\mathrm{pH} 8,150 \mathrm{mM} \mathrm{NaCl}$, at $1 \mathrm{mg} / \mathrm{ml}$ was incubated with nanobody C5, purified in PBS, at a molar ratio of 1:1.2 (Spike monomer:nanobody) at $16^{\circ} \mathrm{C}$ overnight. SPT Labtech prototype 300 mesh 1.2/2.0 nanowire grids were glow-discharged on low for 4 min (Plasma Cleaner PDC-002-CE, Harrick Plasma) and used in a Chameleon EP system (SPT Labtech) at $80 \%$ relative humidity, ambient temperature. Frozen grids were screened, and data collected using Titan Krios G2 (Thermo Fisher Scientific) equipped with a Bioquantum-K3 detector (Gatan, UK) operated at $300 \mathrm{kV}$. Data collection statistics are given in Supplementary Table 3. The RELION_IT.py processing pipeline as implemented in eBIC was used for automatic data processing up to $2 \mathrm{D}$ classification. The data were first processed as $\mathrm{C} 1$ but as the complex showed C3 symmetry, this was later changed to C3. The best 3D class was selected for further refinement, CTF refinement and particle polishing within Relion. An initial model based on PDB ID 6VXX was created and the RBDC5 crystal structure placed into density. The final model with correlation coefficient 0.76 was generated by multiple cycles of manual intervention in $\operatorname{coot}^{56}$ followed by jelly body refinement using RefMac5 via CCP-EM GUI ${ }^{53,54}$. Model validation was carried out in PHENIX ${ }^{62-64}$. Data processing and refinement statistics are given in Table 3.

Microneutralisation assay. VHH trimers were serially diluted into Dulbecco's Modified Eagles Medium (DMEM) containing 1\% (w/v) foetal bovine serum (FBS) in a 96-well plate. SARS-CoV-2 strains (B VIC01, B1.17 and B1.351) passage 4 (Vero 76 clone e6 [ECACC 85020206]) $9 \times 10^{4} \mathrm{pfu} / \mathrm{ml}$ diluted 1:5 in DMEM-FBS were added to each well with media only as negative controls. After incubation for $30 \mathrm{~min}$ at $37^{\circ} \mathrm{C}$, Vero cells $(100 \mu \mathrm{l})$ were added to each well and the plates incubated for $2 \mathrm{~h}$ at $37^{\circ} \mathrm{C}$. Carboxymethyl cellulose $(100 \mu \mathrm{l}$ of $1.5 \% \mathrm{v} / \mathrm{v})$ was then added to each well and the plates incubated for a further $18-20 \mathrm{~h}$ at $37^{\circ} \mathrm{C}$. Cells were fixed with paraformaldehyde $(100 \mu \mathrm{l} /$ well $4 \% \mathrm{v} / \mathrm{v})$ for $30 \mathrm{~min}$ at room temperature and then stained for SARS-CoV-2 nucleoprotein using a human monoclonal antibody (EY2A). Bound antibody was detected by incubation with a goat anti-human IgG HRP conjugate and following substrate addition imaged using an ELISPOT reader. 
The neutralisation titre was defined as the titre of $\mathrm{VHH}$ trimer that reduced the Foci forming unit (FFU) by $50 \%$ compared to the control wells.

PRNT assay. Plaque reduction neutralization tests (PRNT) were carried out at Public Health England using SARS-CoV-2 (hCoV-19/Australia/VIC01/2020) (GISAID accession number EPI_ISL_406844 ${ }^{65}$ generously provided by The Doherty Institute, Melbourne, Australia at P1 and passaged twice in Vero/hSLAM cells [ECACC 04091501]. Virus was diluted to a concentration of 933 p.f.u. $\mathrm{ml}^{-1}$ (70 p.f.u./75 $\mu$ l) and mixed 50:50 in minimal essential medium (MEM; Life Technologies) containing 1\% FBS (Life Technologies) and $25 \mathrm{mM}$ HEPES buffer (Sigma) with doubling antibody dilutions in a 96-well V-bottomed plate. The plate was incubated at $37^{\circ} \mathrm{C}$ in a humidified box for $1 \mathrm{~h}$ to allow neutralisation to take place. Afterwards, the virus-antibody mixture was transferred into the wells of a twice Dulbecco's PBS-washed 24-well plate containing confluent monolayers of Vero E6 cells (ECACC 85020206, PHE) that had been cultured in MEM containing $10 \%(\mathrm{v} / \mathrm{v}) \mathrm{FBS}$. Virus was allowed to adsorb onto cells at $37^{\circ} \mathrm{C}$ for a further hour in a humidified box, then the cells were overlaid with MEM containing $1.5 \%$ carboxymethyl cellulose (Sigma), 4\% (v/v) FBS and 25 mM HEPES buffer. After 5 days incubation at $37^{\circ} \mathrm{C}$ in a humidified box, the plates were fixed overnight with $20 \%$ formalin/PBS $(\mathrm{v} / \mathrm{v})$, washed with tap water and then stained with $0.2 \%$ crystal violet solution (Sigma) and plaques were counted. A mid-point probit analysis (written in $\mathrm{R}$ programming language for statistical computing and graphics) was used to determine the dilution of antibody required to reduce SARS-CoV-2 viral plaques by $50 \%$ (ND50) compared with the virus-only control $(n=5)$. The script used in $\mathrm{R}$ was based on a previously reported source script 44 . Antibody dilutions were run in duplicate and an internal positive control for the PRNT assay was also run in duplicate using a sample of heat-inactivated $\left(56^{\circ} \mathrm{C}\right.$ for $\left.30 \mathrm{~min}\right)$ human MERS convalescent serum pH 7.4, $137 \mathrm{mM} \mathrm{NaCl}, 1 \mathrm{mM} \mathrm{CaCl}_{2}$ ) and $1 \mathrm{mg} \mathrm{ml}^{-1}$ trypsin (Sigma-Aldrich) to neutralise SARS-CoV-2 (National Institute for Biological Standards and Control, UK).

\section{Evaluation of C5-Fc efficacy in the Syrian hamster model (Public Health} England). Golden Syrian hamsters (Mesocricetus auratus) (males and females) aged between 7 and 9 weeks old, weighing 110-140 g, were obtained from Envigo, London, UK. Hamsters were assigned randomly and housed in individual cages with access to food and water ad libitum. All experimental work was conducted under the authority of a UK Home Office approved project license that had been subject to local ethical review at PHE Porton Down by the Animal Welfare and Ethical Review Body (AWERB) as required by the Home Office Animals (Scientific Procedures) Act 1986.

Twelve hamsters were briefly anaesthetised with $5 \%$ isoflurane (Zoetis, Leatherhead, UK) and $4 \mathrm{~L} / \mathrm{m} \mathrm{O} 2$ and inoculated by the intranasal route with $5 \times 10^{4}$ p.f.u/animal of SARS-CoV-2 (hCoV-19/Australia/VIC01/2020) delivered in $100 \mu \mathrm{l}$ per nostril $(200 \mu \mathrm{l}$ in total). At day 1 post-challenge (pc) 6 hamsters were treated with $4 \mathrm{mg} / \mathrm{kg}$ of C5 Nanobody via the intraperitoneal route. Control hamsters $(n=6)$ received no treatment. Temperature (taken using a microchip reader and implanted temperature/ID chip) and clinical signs were monitored twice daily, weight once daily. Clinical signs were scored as follows; healthy $=0$, behavioural changes $=1$, ruffled fur $=2$, wet tail $=2$, dehydrated $=2$, eyes shut $=3$, arched back $=3$, wasp waisted $=3$ and laboured breathing $=5$. Clinical samples of nasal washes in Dulbecco's PBS (DPBS, Gibco) $(200 \mu \mathrm{l})$ as well as oropharyngeal (throat) swabs (MWE, Corsham, UK) were obtained prior to infection (day -2) and on days 2, 4, 6 and $7 \mathrm{pc}$; animals were briefly anaesthetised for the collection of these samples. On day 7 all the hamsters were euthanized by an overdose of anaesthetic (sodium pentobarbitone [Dolelethal, Vetquinol UK Ltd]) via the intraperitoneal route. At necropsy nasal washes and oropharyngeal swabs and tissue samples (lung, trachea and duodenum) were collected in PBS and stored frozen at $-80^{\circ} \mathrm{C}$ for viral RNA measurement and viral culture. Tissue samples for histopathological examination were fixed in $10 \%$ buffered formalin at room temperature (see below)

A micro-plaque assay ${ }^{66}$ was used to determine the amount of virus in tissue samples. The animal sample was serially diluted in assay diluent (MEM

supplemented with L-glutamine (Life Technologies), non-essential amino acids (Life Technologies), $25 \mathrm{mM}$ HEPES (Sigma) and $1 \mathrm{x}$ antibiotic/antimycotic) and added to confluent monolayers of Vero E6 cells. The virus was adsorbed to the cells for $1 \mathrm{hr}$ at $37^{\circ} \mathrm{C}$. The innocula were removed from the cell plates and a viscous overlay ( $1 \%$ carboxymethylcellulose, Sigma) was added. The plates were then incubated for $24 \mathrm{hr}$ at $37^{\circ} \mathrm{C}$. The cells were then fixed using $8 \%$ formalin for $>8 \mathrm{~h}$ and an immunostaining protocol was performed on the fixed cells (Bewley et al.). Stained foci [foci forming units (FFU)] were counted using an ELISpot counter (Cellular Technology Limited, USA). The counted foci data was then plotted using Graph Pad version 9. A SARS-CoV-2 positive control at $1 \times 10^{5} \mathrm{PFU} / \mathrm{ml}$ was run alongside the animal samples, on each assay plate, with uninfected assay diluent as negative control.

RNA was isolated from nasal washes, oropharyngeal swabs and tissue samples (lung, trachea and duodenum). Weighed tissue samples were homogenised and inactivated in RLT (Qiagen) supplemented with $1 \%(\mathrm{v} / \mathrm{v})$ beta-mercaptoethanol. Tissue homogenate was then centrifuged through a QIAshredder homogenizer (Qiagen) and supplemented with ethanol as per manufacturer's instructions. Downstream extraction was then performed using the BioSprint ${ }^{\mathrm{m}} 96$ One-For-All vet kit (Indical Bioscience) and Kingfisher Flex platform as per manufacturer's instructions. Non-tissue samples were inactivated in AVL (Qiagen) and ethanol, with final extraction using the BioSprint ${ }^{\mathrm{m}} 96$ One-For-All vet kit (Indical Bioscience) and Kingfisher Flex platform as per manufacturer's instructions. Reverse transcription-quantitative polymerase chain reaction (RT-qPCR) was performed using TaqPath ${ }^{\text {mx }} 1$-Step RT-qPCR Master Mix, CG (Applied Biosystems $s^{\text {ntw }}$ ), 2019-nCoV CDC RUO Kit (Integrated DNA Technologies) and QuantStudio $^{\text {max }} 7$ Flex Real-Time PCR System. Sequences of the N1 primers and probe were: 2019-nCoV_N1-forward, 5' GACCCCAAAATCAGCGAAAT 3'; 2019-nCoV_N1-reverse, $5^{\prime}$ TCTGGTTACTGCCAGTTGAATCTG 3'; 2019nCoV_N1-probe, 5' FAM-ACCCCGCATTACGTTTGGTGGACC-BHQ1 $3^{\prime}$. The cycling conditions were $25^{\circ} \mathrm{C}$ for $2 \mathrm{~min}, 50^{\circ} \mathrm{C}$ for $15 \mathrm{~min}, 95^{\circ} \mathrm{C}$ for $2 \mathrm{~min}$, followed by 45 cycles of $95^{\circ} \mathrm{C}$ for $3 \mathrm{~s}, 55^{\circ} \mathrm{C}$ for $30 \mathrm{~s}$. The quantification standard was in vitro transcribed RNA of the SARS-CoV-2 N ORF (accession number NC_045512.2) with quantification between 10 and $1 \times 10^{6}$ copies/ $\mu$ l. Positive samples detected below the lower limit of quantification (LLOQ) of 10 copies/ $\mu$ l were assigned the value of 5 copies $/ \mu l$, undetected samples were assigned the value of $2.3 \mathrm{copies} / \mu \mathrm{l}$, equivalent to the assays LLOD. For nasal wash and oropharyngeal swab extracted samples this equates to an LLOQ of $1.29 \times 10^{4}$ copies $/ \mathrm{mL}$ and LLOD of $2.96 \times 10^{3}$ copies $/ \mathrm{mL}$. Samples detected between LLOQ and LLOD were assigned $6.43 \times 10^{3}$ copies $/ \mathrm{mL}$. For tissue samples this equates to an LLOQ of $1.31 \times 10^{4}$ copies $/ \mathrm{g}$ and LLOD of $5.71 \times 10^{4}$ copies/g. Samples detected between LLOQ and LLOD were assigned $2.86 \times 10^{4}$ copies $/ \mathrm{g}$.

Subgenomic RT-qPCR was performed on the QuantStudio 7 Flex Real-Time PCR System using TaqMan ${ }^{\text {nt }}$ Fast Virus 1-Step Master Mix (Thermo Fisher Scientific) and oligonucleotides as specified by Wolfel et al. ${ }^{67}$, with forward primer, probe and reverse primer at a final concentration of $250 \mathrm{nM}, 125 \mathrm{nM}$ and $500 \mathrm{nM}$ respectively. Sequences of the $\mathrm{sgE}$ primers and probe were: $2019-\mathrm{nCoV} s \mathrm{sgE}-$ forward, 5' CGATCTCTTGTAGATCTGTTCTC 3'; 2019-nCoV_sgE-reverse, 5 ATATTGCAGCAGTACGCACACA $3^{\prime}$ and 2019-nCoV_sgE-probe, $5^{\prime}$ FAMACACTAGCCATCCTTACTGCGCTTCG-BHQ1 $3^{\prime}$. Cycling conditions were $50{ }^{\circ} \mathrm{C}$ for $10 \mathrm{~min}, 95^{\circ} \mathrm{C}$ for $2 \mathrm{~min}$, followed by 45 cycles of $95^{\circ} \mathrm{C}$ for $10 \mathrm{~s}$ and $60^{\circ} \mathrm{C}$ for $30 \mathrm{~s}$. RT-qPCR amplicons were quantified against an in vitro transcribed RNA standard of the full-length SARS-CoV-2 E ORF (accession number NC_045512.2) preceded by the UTR leader sequence and putative $\mathrm{E}$ gene transcription regulatory sequence described by Wolfel et al. in 202049. Positive samples detected below the lower limit of quantification (LLOQ) were assigned the value of 5 copies $/ \mu$, whilst undetected samples were assigned the value of $\leq 0.9$ copies/ $\mu$ l, equivalent to the lower limit of detection of the assay (LLOD). For nasal washes and oropharyngeal swabs extracted samples this equated to an LLOQ of $1.29 \times 10^{4}$ copies $/ \mathrm{mL}$ and LLOD of $1.16 \times 10^{3}$ copies $/ \mathrm{mL}$. For tissue samples this equates to an LLOQ of $5.71 \times 10^{4}$ copies/g and LLOD of $5.14 \times 10^{3}$ copies/g.

The lung, nasal cavity including olfactory and respiratory mucosa, heart, liver, spleen, pancreas, trachea/larynx brain and small intestine (duodenum) were taken from each animal and were fixed in $10 \%$ neutral-buffered formalin, processed, embedded in paraffin wax and $4 \mu \mathrm{m}$ thick sections cut and stained with haematoxylin and eosin (H\&E). The tissue sections were digitally scanned and reviewed by a qualified veterinary pathologist blinded to treatment and group details and the slides were randomised prior to examination in order to prevent bias (blind evaluation). A scoring system was used to evaluate objectively the histopathological lesions observed in the tissue sections: $0=$ within normal limits; $1=$ minimal; $2=$ mild; $3=$ moderate and $4=$ marked $/$ severe. Moreover, the area of the lung with pneumonia was calculated using digital image analysis (NikonNIS-Ar software package).

RNAscope (an in-situ hybridisation method used on formalin-fixed, paraffinembedded tissues) was used to identify the SARS-CoV-2 virus in all tissues. Briefly, tissues were pre-treated with hydrogen peroxide for 10 mins at room temperature (RT) target retrieval for $15 \mathrm{mins}\left(98-101^{\circ} \mathrm{C}\right)$ and protease plus for $30 \mathrm{mins}\left(40^{\circ} \mathrm{C}\right)$ (all Advanced Cell Diagnostics). A V-nCoV2019-S probe (Advanced Cell Diagnostics) targeting the S-protein gene was incubated on the tissues for $2 \mathrm{~h}$ at $40^{\circ} \mathrm{C}$. Amplification of the signal was carried out following the RNAscope protocol (RNAscope 2.5 HD Detection Reagent - Red) using the RNAscope 2.5 HD red kit (Advanced Cell Diagnostics). Appropriate controls were included in each ISH run. Digital image analysis was carried out with the Nikon NIS-Ar software package in order to calculate the total area of the tissue section positive for viral RNA. The images were scanned digitally using a Hamamatsu NanoZoomer S360 digital slide scanner and examined using Ndp.view2 v2.9.22 software. Nikon NIS-Ar software was used to perform digital image analysis in order to quantify the presence of viral RNA in lung sections. Graph and statistical analysis were performed with Graphpad Prism 9 and Minitab version 16.

\section{Evaluation of C5 trimer therapeutic efficacy in the Syrian hamster model} (University of Liverpool). Animal work was approved by the local University of Liverpool Animal Welfare and Ethical Review Body and performed under UK Home Office Project Licence PP4715265. Male golden Syrian hamsters (8-10 weeks old) were purchased from Janvier Labs (France). Animals were maintained under SPF barrier conditions in individually ventilated cages. For virus infection the Liverpool strain was used, a PANGO lineage B strain of SARS-CoV-2 (hCoV-2/human/Liverpool/REMRQ0001/2020) ${ }^{68}$. Animals were randomly assigned into multiple cohorts of 6 animals. For SARS-CoV-2 infection, hamsters 
were anaesthetised lightly with isoflurane and inoculated intranasally with $100 \mu$ containing $10^{4}$ PFU SARS-CoV-2 in PBS. Hamsters were treated with $100 \mu \mathrm{l}$ via either the intraperitoneal or intranasal route with C5 trimer contained in PBS. Animals were killed at variable time-points after infection by an overdose of pentabarbitone. Tissues were removed immediately for downstream processing.

From all animals the left lung was fixed in $10 \%$ buffered formalin for $48 \mathrm{~h}$ and then stored in $70 \%$ ethanol until further processing. Two longitudinal sections were prepared and routinely paraffin wax embedded. Consecutive sections $(3-5 \mu \mathrm{m})$ were prepared and stained with HE for histological examination or subjected to immunohistological staining. Immunohistology was performed to detect SARSCoV-2 antigen, macrophages (Iba1+), type II pneumocytes (SP-C+) and epithelial cells (pan-cytokeratin + ), using the horseradish peroxidase (HRP) method and the following primary antibodies: rabbit anti-SARS-CoV nucleocapsid protein (1:6000, Rockland, 200-402-A50), rabbit anti-human Iba1/AIF1 (1:1000, Wako, 019-19741), rabbit anti-human prosurfactant protein-C (1:4000, SP-C; Abcam, ab40879) and mouse anti-human pan-cytokeratin (1:10000, clone PCK-26; Novus Biologicals, NB120-6401). Briefly, after de-paraffination, sections underwent antigen retrieval in citrate buffer (pH 6.0; Agilent) (anti-SARS-CoV-2, -Iba1) or Tris-EDTA buffer (pH 9.0) (anti-SP-C, -pan-cytokeratin) for $20 \mathrm{~min}$ at $98^{\circ} \mathrm{C}$ and for $20 \mathrm{~min}$ at $37^{\circ} \mathrm{C}$ respectively, followed by incubation with the primary antibody overnight at $4{ }^{\circ} \mathrm{C}$ (anti-SARS-CoV, SP-C) or $60 \mathrm{~min}$ at RT (anti-Ibal, -pan-cytokeratin). This was followed by blocking of endogenous peroxidase (peroxidase block, Agilent) for $10 \mathrm{~min}$ at room temperature (RT) and incubation with the secondary antibody, EnVision+/HRP, Rabbit and Mouse respectively (undiluted ready-to-use reagent, Agilent K406311-2) for 30 min at RT, followed by EnVision FLEX

$\mathrm{DAB}+$ Chromogen in Substrate buffer (Agilent) for $10 \mathrm{~min}$ at RT, all in an autostainer (Dako). Sections were subsequently counterstained with haematoxylin. The anti-Ibal, -SP-C and -pan-cytokeratin antibodies were tested for their cross reactivity in hamster tissues, using the lung of an uninfected control hamster as positive control.

For double immunofluorescence, sections underwent antigen retrieval in citrate buffer $(\mathrm{pH}$ 6.0) and were then incubated with the first primary antibody (rabbit anti-SARS-CoV), overnight at $4{ }^{\circ} \mathrm{C}$, followed by blocking of the endogenous peroxidase (see above) and $1 \mathrm{~h}$ incubation with the red fluorescence labelled antibody (1:500, goat anti-rabbit 594; Invitrogen, A11012), incubation with the second primary antibody (1:400, goat anti-human Iba1; Abcam, ab 5076), overnight at $4^{\circ} \mathrm{C}$, and $1 \mathrm{~h}$ incubation with the green fluorescence labelled antibody (1:500, donkey anti-goat 488; Invitrogen, A1105). The final incubation was with DAPI (4', 6-diamidino-2-phenylindole, Novus Biologicals), for $15 \mathrm{~min}$ at RT. After that, sections were washed twice with distilled water, air dried, and a coverslip placed with FluoreGuard mounting medium (Biosystems, Switzerland).

For morphometric analysis, the HE-stained sections were scanned (NanoZoomer-XR C12000; Hamamatsu, Hamamatsu City, Japan) and analysed using the software programme Visiopharm (Visiopharm 2020.08.1.8403; Visiopharm, Hoersholm, Denmark) to quantify the area of non-aerated parenchyma and aerated parenchyma in relation to the total area (= area occupied by lung parenchyma on two sections prepared from the left lung lobes) in the sections. This was used to compare the amount of air space (as an equivalent for the gas exchange surface) in the lungs between untreated and treated animals. A first app was applied that outlined the entire lung tissue as Region Of Interest (ROI, total area). For this a Decision forest method was used and the software was trained to detect the lung tissue section (total area). Once the lung section was outlined as ROI the large bronchi and vessels were manually excluded from the ROI. Subsequently, a second app with Decision forest method was trained to detect dense parenchyma (non-ventilated) and alveolar spaces (clear spaces; ventilated area) within the ROI.

Reporting summary. Further information on research design is available in the Nature Research Reporting Summary linked to this article.

\section{Data availability}

The coordinates and structure factors were deposited in the wwPDB with accession nos. C5 - RBD 7OAO, H3- RBD-C1 7OAP, F2-RBD 7OAY, C5-Alpha-RBD 7OAU and H3Alpha RBD-C1 7OAU. Spike C5 EM maps and models are deposited in the EMDB and wwPDB under accession codes, EMD-12777 and 7OAN. Nanobody sequences are provided in the Supplementary Table 2. Source data are provided with this paper.

Received: 27 May 2021; Accepted: 12 August 2021;

Published online: 22 September 2021

\section{References}

1. Dhama, K. et al. Coronavirus disease 2019-COVID-19. Clin. Microbiol. Rev. https://doi.org/10.1128/CMR.00028-20 (2020).

2. Zost, S. J. et al. Potently neutralizing and protective human antibodies against SARS-CoV-2. Nature 584, 443-449 (2020).
3. Liu, L. et al. Potent neutralizing antibodies against multiple epitopes on SARS CoV-2 spike. Nature 584, 450-456 (2020).

4. Ju, B. et al. Human neutralizing antibodies elicited by SARS-CoV-2 infection. Nature 584, 115-119 (2020).

5. Cao, Y. et al. Potent neutralizing antibodies against SARS-CoV-2 identified by high-throughput single-cell sequencing of convalescent patients' B cells. Cell 182, 73-84 e16 (2020).

6. Rogers, T. F. et al. Isolation of potent SARS-CoV-2 neutralizing antibodies and protection from disease in a small animal model. Science 369, 956-963 (2020).

7. Chen, F., Liu, Z. \& Jiang, F. Prospects of neutralizing nanobodies against SARS-CoV-2. Front. Immunol. 12, 690742 (2021).

8. Huo, J. et al. Neutralizing nanobodies bind SARS-CoV-2 spike RBD and block interaction with ACE2. Nat. Struct. Mol. Biol. 27, 846-854 (2020).

9. Wrapp, D. et al. Structural basis for potent neutralization of betacoronaviruses by single-domain camelid antibodies. Cell 181, 1436-1441 (2020).

10. Schoof, M. et al. An ultrapotent synthetic nanobody neutralizes SARS-CoV-2 by stabilizing inactive Spike. Science 370, 1473-1479 (2020).

11. Koenig, P. A. et al. Structure-guided multivalent nanobodies block SARSCoV-2 infection and suppress mutational escape. Science https://doi.org/ 10.1126/science.abe6230 (2021)

12. Custodio, T. F. et al. Selection, biophysical and structural analysis of synthetic nanobodies that effectively neutralize SARS-CoV-2. Nat. Commun. 11, 5588 (2020).

13. Xiang, Y. et al. Versatile and multivalent nanobodies efficiently neutralize SARS-CoV-2. Science 370, 1479-1484 (2020).

14. Hanke, L. et al. An alpaca nanobody neutralizes SARS-CoV-2 by blocking receptor interaction. Nat. Commun. 11, 4420 (2020).

15. Xu, J. et al. Nanobodies from camelid mice and llamas neutralize SARS-CoV-2 variants. Nature https://doi.org/10.1038/s41586-021-03676-z (2021).

16. Valenzuela Nieto, G. et al. Potent neutralization of clinical isolates of SARSCoV-2 D614 and G614 variants by a monomeric, sub-nanomolar affinity nanobody. Sci. Rep. 11, 3318 (2021).

17. Pymm, P. et al. Nanobody cocktails potently neutralize SARS-CoV-2 D614G N501Y variant and protect mice. Proc. Natl Acad. Sci. USA https://doi.org/ $10.1073 /$ pnas.2101918118 (2021).

18. Nambulli, S. et al. Inhalable Nanobody (PiN-21) prevents and treats SARSCoV-2 infections in Syrian hamsters at ultra-low doses. Sci. Adv. https:// doi.org/10.1126/sciadv.abh0319 (2021).

19. Cardoso, F. M. et al. Single-domain antibodies targeting neuraminidase protect against an H5N1 influenza virus challenge. J. Virol. 88, 8278-8296 (2014).

20. Richard, G. et al. In vivo neutralization of alpha-cobratoxin with high-affinity llama single-domain antibodies (VHHs) and a VHH-Fc antibody. PLoS ONE 8, e69495 (2013).

21. Rotman, M. et al. Fusion of hIgG1-Fc to 111In-anti-amyloid single domain antibody fragment VHH-pa2H prolongs blood residential time in APP/PS1 mice but does not increase brain uptake. Nucl. Med. Biol. 42, 695-702 (2015).

22. Els Conrath, K., Lauwereys, M., Wyns, L. \& Muyldermans, S. Camel singledomain antibodies as modular building units in bispecific and bivalent antibody constructs. J. Biol. Chem. 276, 7346-7350 (2001).

23. Detalle, L. et al. Generation and characterization of ALX-0171, a potent novel therapeutic nanobody for the treatment of respiratory syncytial virus infection. Antimicrob. Agents Chemother. 60, 6-13 (2016).

24. Jan ter Meulen Edward, N. et al. Human monoclonal antibody combination against SARS coronavirus: synergy and coverage of escape mutants. PLoS Med. 3, e237 (2006)

25. Huo, J. et al. Neutralizing nanobodies bind SARS-CoV-2 spike RBD and block interaction with ACE2. Nat. Struct. Mol. Biol. 27, 846-885 (2020).

26. Huo, J. et al. Neutralization of SARS-CoV-2 by destruction of the prefusion spike. Cell Host Microbe. 28, 445-454 (2020).

27. Yuan, M. et al. Structural basis of a shared antibody response to SARS-CoV-2. Science 369, 1119-1123 (2020).

28. HuangI, Kuan-YingA. et al. Breadth and function of antibody response to acute SARS-CoV-2 infection in humans. PLoS Pathog. 17, e1009352 (2021).

29. Zhou, D. et al. Structural basis for the neutralization of SARS-CoV-2 by an antibody from a convalescent patient. Nat. Struct. Mol. Biol. 27, 950-958 (2020).

30. Daming Zhou, W. D. et al. Evidence of escape of SARS-CoV-2 variant B.1.351 from natural and vaccine induced sera. Cell https://doi.org/10.1016/ j.cell.2021.02.037 (2021)

31. Piyada Supasa, D. Z. et al. Screaton. Reduced neutralization of SARS-CoV-2 B.1.1.7 variant by convalescent and vaccine sera. Cell https://doi.org/10.1016/ j.cell.2021.02.033 (2021).

32. Luan, B., Wang, H. \& Huynh, T. Enhanced binding of the N501Y-mutated SARS-CoV-2 spike protein to the human ACE2 receptor: insights from molecular dynamics simulations. FEBS Lett. 595, 1454-1461 (2021). 
33. Greaney, A. J. et al. Complete mapping of mutations to the SARS-CoV-2 spike receptor-binding domain that escape antibody recognition. Cell Host Microbe 29, 44-57 e49 (2021).

34. Wrapp, D. et al. Cryo-EM structure of the 2019-nCoV spike in the prefusion conformation. Science 367, 1260-1263 (2020).

35. Walls, A. C. et al. Structure, function, and antigenicity of the SARS-CoV-2 spike glycoprotein. Cell 181, 281-292 e286 (2020).

36. Wrobel, A. G. et al. SARS-CoV-2 and bat RaTG13 spike glycoprotein structures inform on virus evolution and furin-cleavage effects. Nat. Struct. Mol. Biol. 27, 763-767 (2020).

37. He, L. et al. Enhanced ability of oligomeric nanobodies targeting MERS coronavirus receptor-binding domain. Viruses https://doi.org/10.3390/ v11020166 (2019).

38. Hultberg, A. et al. Llama-derived single domain antibodies to build multivalent, superpotent and broadened neutralizing anti-viral molecules. PLoS ONE 6, e17665 (2011).

39. Koenig, P. A. et al. Structure-guided multivalent nanobodies block SARS CoV-2 infection and suppress mutational escape. Science https://doi.org/ 10.1126/science.abe6230 (2021)

40. Kondo, T. et al. Antibody-like proteins that capture and neutralize SARS CoV-2. Sci. Adv. https://doi.org/10.1126/sciadv.abd3916 (2020).

41. Chan, J. F. et al. Simulation of the clinical and pathological manifestations of Coronavirus Disease 2019 (COVID-19) in a golden syrian hamster model: implications for disease pathogenesis and transmissibility. Clin. Infect. Dis. 71, 2428-2446 (2020).

42. Imai, M. et al. Syrian hamsters as a small animal model for SARS-CoV-2 infection and countermeasure development. Proc. Natl Acad. Sci. USA 117, 16587-16595 (2020).

43. Sia, S. F. et al. Pathogenesis and transmission of SARS-CoV-2 in golden hamsters. Nature 583, 834-838 (2020).

44. Roberts, A. et al. Severe acute respiratory syndrome coronavirus infection of golden Syrian hamsters. J. Virol. 79, 503-511 (2005).

45. Zhao, G. et al. A novel nanobody targeting middle east respiratory syndrome coronavirus (MERS-CoV) receptor-binding domain has potent crossneutralizing activity and protective efficacy against MERS-CoV. J. Virol. https://doi.org/10.1128/JVI.00837-18 (2018).

46. Liang, W., Pan, H. W., Vllasaliu, D. \& Lam, J. K. W. Pulmonary delivery of biological drugs. Pharmaceutics https://doi.org/10.3390/ pharmaceutics12111025 (2020).

47. Yuan, M. et al. A highly conserved cryptic epitope in the receptor binding domains of SARS-CoV-2 and SARS-CoV. Science 368, 630-633 (2020).

48. Wrapp, D. et al. Structural basis for potent neutralization of betacoronaviruses by single-domain camelid antibodies. Cell 181, 1004-1015.e1015 (2020).

49. Van Heeke, G. et al. Nanobodies(R) as inhaled biotherapeutics for lung diseases. Pharmacol. Ther. 169, 47-56 (2017).

50. Esparza, T. J., Martin, N. P., Anderson, G. P., Goldman, E. R. \& Brody, D. L. High affinity nanobodies block SARS-CoV-2 spike receptor binding domain interaction with human angiotensin converting enzyme. Sci. Rep. 10, 22370 (2020).

51. Nettleship, J. E., Rahman-Huq, N. \& Owens, R. J. The production of glycoproteins by transient expression in Mammalian cells. Methods Mol. Biol. 498, 245-263 (2009).

52. Bird, L. E. et al. Application of In-Fusion cloning for the parallel construction of E. coli expression vectors. Methods Mol. Biol. 1116, 209-234 (2014).

53. McCoy, A. J., Grosse-Kunstleve, R. W., Storoni, L. C. \& Read, R. J. Likelihoodenhanced fast translation functions. Acta Crystallogr D Biol. Crystallogr. 61, 458-464 (2005).

54. Storoni, L. C., McCoy, A. J. \& Read, R. J. Likelihood-enhanced fast rotation functions. Acta Crystallogr. D Biol. Crystallogr. 60, 432-438 (2004).

55. Winn, M. D. et al. Overview of the CCP4 suite and current developments. Acta Crystallogr. D Biol. Crystallogr. 67, 235-242 (2011).

56. Emsley, P. \& Cowtan, K. Coot: model-building tools for molecular graphics. Acta Crystallogr. D Biol. Crystallogr. 60, 2126-2132 (2004).

57. Murshudov, G. N. et al. REFMAC5 for the refinement of macromolecular crystal structures. Acta Crystallogr. D Biol. Crystallogr. 67, 355-367 (2011).

58. Joosten, R. P., Long, F., Murshudov, G. N. \& Perrakis, A. The PDB_REDO server for macromolecular structure model optimization. IUCrJ 1, 213-220 (2014).

59. Davis, I. W. et al. MolProbity: all-atom contacts and structure validation for proteins and nucleic acids. Nucleic Acids Res. 35, W375-W383 (2007).

60. Winn, M. D., Murshudov, G. N. \& Papiz, M. Z. Macromolecular TLS refinement in REFMAC at moderate resolutions. Methods Enzymol. 374, 300-321 (2003).

61. Painter, J. M. \& E., A. TLSMD web server for the generation of multi-group TLS models. J. Appl. Crystallogr. 39, 109-111 (2006).
62. Liebschner, D. et al. Macromolecular structure determination using X-rays, neutrons and electrons: recent developments in Phenix. Acta Crystallogr. D Struct. Biol. 75, 861-877 (2019).

63. Williams, C. J. et al. MolProbity: More and better reference data for improved all-atom structure validation. Protein Sci. 27, 293-315 (2018).

64. Afonine, P. V. et al. New tools for the analysis and validation of cryo-EM maps and atomic models. Acta Crystallogr. D Struct. Biol. 74, 814-840 (2018).

65. Caly, L. et al. Isolation and rapid sharing of the 2019 novel coronavirus (SARS-CoV-2) from the first patient diagnosed with COVID-19 in Australia. Med. J. Aust. 212, 459-462 (2020).

66. Bewley, K. R. et al. Quantification of SARS-CoV-2 neutralizing antibody by wild-type plaque reduction neutralization, microneutralization and pseudotyped virus neutralization assays. Nat. Protoc. 16, 3114-3140 (2021).

67. Wolfel, R. et al. Virological assessment of hospitalized patients with COVID2019. Nature 581, 465-469 (2020)

68. Patterson, E. I. et al. Methods of inactivation of SARS-CoV-2 for downstream biological assays. J. Infect. Dis. 222, 1462-1467 (2020).

\section{Acknowledgements}

This work was supported by the Rosalind Franklin Institute, funding delivery partner EPSRC. PPUK is funded by the Rosalind Franklin Institute EPSRC grant no. EP/ S025243/1. J.H.N., A.L.B., P.J.H., M.W. and P.W. are supported by Wellcome Trust (100209/Z/12/Z). J.H. is supported by the EPA Cephalosporin Fund. X-ray data were obtained using Diamond Light Source COVID-19 Rapid Access time on Beamline I03, I04 and I24 (proposal MX27031). The core virus neutralisation facility is supported by gifts to the Oxford COVID-19 Research Response Fund. EM results were obtained at the national EM facility at Diamond, eBIC, through rapid access proposal BI27051. Work at the University of Liverpool is supported by MRC grant MR/W005611/1, G2P-UK; A National Virology Consortium to address phenotypic consequences of SARSCoV-2 genomic variation (JPS and JAH) and by the US Food and Drug Administration (USA) 75F40120C00085, Characterisation of severe coronavirus infection in humans and model systems for medical countermeasure development and evaluation (JAH). We wish to thank the laboratory staff of the Histology Laboratory, Institute of Veterinary Pathology, Vetsuisse Faculty, University of Zurich, and the laboratory staff of the Pathology Laboratory and Biological Investigations Group Public Health England, Porton Down for excellent technical support. We are grateful to Josep Monné Rodriguez for his assistance in the design of the apps for the morphometric assessment. We thank Tomas Malinauskas (Oxford University) and colleagues at the CMB (Oxford University) for assistance with protein production and Professor Gary Stephens, Barney Jones and Hong Lin (Reading University) for expertise in llama immunisation.

\section{Author contributions}

J.H. isolated the nanobodies, designed trimers and carried out SPR analyses. M.W. and D.K.C. performed the EM studies. H.M., L.M., A.L.B., J.H. and J.H.N. performed the crystallography and ITC experiments. J.H., A.L.B., J.D., C.N., P.J.H., P.N.W. and M.D. produced proteins for the experiments. A.H., K.R.B., M.J.E. and W.J. carried out neutralization assays and analysis. J.J.C., P.S., Y.H. and S.F. carried out the animal study. R.W., O.C., D.K., D.N. and T.P. carried out the molecular biology and live viral assays A.K. and F.J.S. performed pathological analyses, immunohistology and morphometric analyses. J.P.S., A.O., J.A.H., J.A.T. and M.W.C. directed the animal studies. R.J.O. and J.H.N. planned the project and wrote the manuscript with contributions from all authors.

\section{Competing interests}

The Rosalind Franklin Institute has filed a patent that includes the four nanobodies described here, R.J.O., J.H. and J.H.N. are named as inventors. The other authors declare no competing interests.

\section{Additional information}

Supplementary information The online version contains supplementary material available at https://doi.org/10.1038/s41467-021-25480-z.

Correspondence and requests for materials should be addressed to James H. Naismith or Raymond J. Owens.

Peer review information Nature Communications thanks Wai-Hong Tham and the other, anonymous, reviewer(s) for their contribution to the peer review of this work. Peer reviewer reports are available.

Reprints and permission information is available at http://www.nature.com/reprints

Publisher's note Springer Nature remains neutral with regard to jurisdictional claims in published maps and institutional affiliations. 
(c) (i) Open Access This article is licensed under a Creative Commons Attribution 4.0 International License, which permits use, sharing, adaptation, distribution and reproduction in any medium or format, as long as you give appropriate credit to the original author(s) and the source, provide a link to the Creative Commons license, and indicate if changes were made. The images or other third party material in this article are included in the article's Creative Commons license, unless indicated otherwise in a credit line to the material. If material is not included in the article's Creative Commons license and your intended use is not permitted by statutory regulation or exceeds the permitted use, you will need to obtain permission directly from the copyright holder. To view a copy of this license, visit http://creativecommons.org/ licenses/by/4.0/.

(C) The Author(s) 2021 